\title{
Enantioselective Synthesis of
}

\section{Trifluoromethyl-substituted Cyclopropanes.}

\author{
Justin R. Denton, Dinesh Sukuaran, and Huw M. L. Davies* \\ ${ }^{*}$ Department of chemistry, University at Buffalo, The state University of New York, Buffalo, NY \\ $14260-300$
}

Supporting Information

Experimental Procedures

S-2 - S-11

${ }^{19} \mathrm{~F}\left\{{ }^{1} \mathrm{H}\right\}$ HOESY NMR Experimental Details

S-12

X-ray Analysis: Synthesis and Experimental Details

S-13 - S-14

Spectral Data

S-15 - S- 32 
General: Reagents were purchased at the highest commercial quality and used without further purification unless otherwise stated. Analytical thin layer chromatography was performed on Whatman silica gel plates using UV light unless stated otherwise. Flash column chromatography was carried out using E. Merck silica $60 .{ }^{1} \mathrm{H}$ NMR spectra were recorded on a Varian Nuclear Magnetic Resonance spectrometer at 300, 400, or 500 MHz. Data are presented as follows: chemical shift (in ppm on the $\delta$ scale relative to $\delta H$ 0.00 for the residual protons in TMS $)$, multiplicity $(\mathrm{s}=$ singlet, $\mathrm{d}=$ doublet, $\mathrm{t}=$ triplet, $\mathrm{q}=$ quartet, $\mathrm{m}=$ multiplet, $\mathrm{dd}=$ doublet of doublets), coupling constant $(\mathrm{J} / \mathrm{Hz})$, and intergration. ${ }^{13} \mathrm{C}$ spectra were recorded at 75.45 or $125 \mathrm{MHz}$ and all chemical shift values are reported in ppm on the $\delta$ scale, with an internal reference of $\delta \mathrm{C} 77.0$ for $\mathrm{CDCl}_{3}$. ${ }^{19}$ NMR spectra were recorded on a Varian Nuclear Magnetic Resonance spectrometer at $375 \mathrm{MHz}$, with an internal reference of TMS. Infrared spectra were obtained on a Nicolet Impact 420 FT-IR spectrometer. Mass spectra were recorded on a Hewlett-Packard GCMS system using $70 \mathrm{eV}$ electron impact (EI) ionization or carried out by LC-MS (ESI). High-resolution mass spectra were obtained from the Mass Spectrometry Facility at the University at Buffalo, The State University of New York. Elemental analyses were determined by Atlantic Microlab, Inc., Norcross, GA. Melting points are uncorrected and optical rotations were measured at the sodium D line $(589 \mathrm{~nm})$ and reported as follows: $[\alpha]_{\mathrm{D}}^{25}$, concentration $(c$ in $\mathrm{g} / 100 \mathrm{~mL})$, and solvent $\left(\mathrm{CHCl}_{3}\right)$. Enantiomeric excess was determined by high performance liquid chromatography (HPLC) using Chiralcel OJ chiral analytical column (UV detection at $230 \mathrm{~nm}$ ) unless otherwise stated. All moisture sensitive reactions were performed using glassware flame dried under vacuum and purged with argon prior to use. Solvents used in rhodium carbenoid transformations were degassed with argon for 10-15 min. prior to use. $\mathrm{Rh}_{2}(S \text {-PTTL })_{4}$ was synthesized from the literature procedure ${ }^{1}$ and used without additional purification. $\mathrm{Rh}_{2}(S \text {-DOSP })_{4}$ and $\mathrm{Rh}_{2}(S$ PTAD) $)_{4}$ are both commercially available from $\mathrm{STREM}^{\circledR}$.

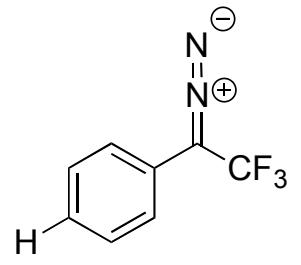

9

\section{1-Phenyl-2,2,2-trifluorodiazoethane (9):}

The diazo compound (9) was synthesized via a modified literature procedure. ${ }^{2} \mathrm{KOH}$ $(11.6 \mathrm{mmol}, 0.650 \mathrm{~g})$ was dissolved in $\mathrm{MeOH}(15 \mathrm{~mL})$ by stirring at room temperature. 4-Methyl- $N$ '-(2,2,2-trifluoro-1-phenylethylidene)benzenesulfonohydrazide (11) (5.8 mmol, $2.00 \mathrm{~g}$ ) was added in one portion and the contents were heated to reflux for $\sim 1 \mathrm{~h}$ and then cooled by addition of water $(15 \mathrm{~mL})$. The aqueous layer was then extracted with dichloromethane (DCM), dried over $\mathrm{MgSO}_{4}$, filtered, and concentrated in vacuo. The orange residue was further purified by flash chromatography (silica gel, pentane) to give $9\left(0.442 \mathrm{~g}, \sim 40 \%\right.$ yield with trace amounts of pentane). ${ }^{1} \mathrm{H} \mathrm{NMR}\left(400 \mathrm{MHz}, \mathrm{CDCl}_{3}\right) \delta$

\footnotetext{
${ }^{1}$ Tsutsui, H.; Abe, T.; Nakamura, S.; Anada, M.; Hashimoto, S. Chem. Pharm. Bull. 2005, 53, 1366.

${ }^{2}$ Shepard, R. A., Wentworth, S. E. J.Org. Chem. 1967, 32, 3197.
} 
7.42-7.38 (m, 2H), 7.22-7.18 (m, 1H), $7.10(\mathrm{~d}, J=8.4 \mathrm{~Hz}, 2 \mathrm{H})$. The ${ }^{1} \mathrm{H}$ NMR data were consistent with the published values. ${ }^{1}$

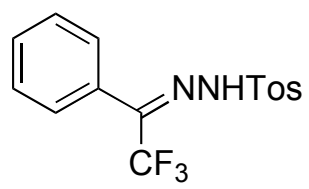

11

4-Methyl- $N$ '-(2,2,2-trifluoro-1-phenylethylidene)benzenesulfonohydrazide (11): $2,2,2$-Trifluoroacetophenone $(2.60 \mathrm{mmol}, 0.448 \mathrm{~g})$ was added to ethanol $(5 \mathrm{~mL})$ at room temperature and $p$-toluenesulfon hydrazide $(2.20 \mathrm{mmol}, 0.410 \mathrm{~g})$ was added in one portion. The contents were then stirred for $\sim 10 \mathrm{~min}$. and 2 drops of acetic acid were added. A condenser was then attached and the contents were heated to reflux. After $\sim 8 \mathrm{~h}$ at reflux, the contents were cooled to room temperature with stirring and then the hydrazone was precipitated by adding distilled water $(\sim 10 \mathrm{~mL})$. The white solid was collected by vacuum filtration and was recrystallized from boiling absolute ethanol to give $11(0.612 \mathrm{~g}$, yield $82 \%)$. ${ }^{1} \mathrm{H}$ NMR $\left(400 \mathrm{MHz}, \mathrm{CDCl}_{3}\right) \delta 7.92(\mathrm{~s}, 1 \mathrm{H}), 7.82(\mathrm{~d}, J=$ $7.6 \mathrm{~Hz}, 2 \mathrm{H}), 7.55-7.53(\mathrm{~m}, 3 \mathrm{H}), 7.37$ (d, $J=7.2 \mathrm{~Hz}, 2 \mathrm{H}), 7.26-7.24(\mathrm{~m}, 2 \mathrm{H}), 2.48(\mathrm{~s}, 3 \mathrm{H})$. The ${ }^{1} \mathrm{H}$ NMR data were consistent with the published values ${ }^{1}$.

\section{General Procedure for $=\mathrm{N}-\mathrm{NH}_{2}$ hydrazone formation:}

The following procedure is typical for all of the $\mathrm{NH}_{2}$ hydrazone formation reactions. Trifluoromethyl ketone $(1.05 \mathrm{mmol})$ was added to absolute ethanol $(\sim 5 \mathrm{~mL})$ and stirred at room temperature. Anhydrous hydrazine $(1.00 \mathrm{mmol})$ was then slowly added drop wise and stirred for $\sim 5 \mathrm{~min}$. Acetic acid ( 2 drops) was then added and a condenser was then attached. The contents were then refluxed overnight $(\sim 12 \mathrm{hr})$. Contents were then cooled to room temperature. At room temperature water $(10 \mathrm{~mL})$ was added and the solution was extracted with diethyl ether $(3 \mathrm{X} 10 \mathrm{~mL})$. The organic layers were combined, dried over $\mathrm{MgSO}_{4}$, filtered, and then concentrated at reduced pressure. The crude hydrazone was then purified by column chromatography (silica gel, diethyl ether) at a fast rate to reduce product decomposition.

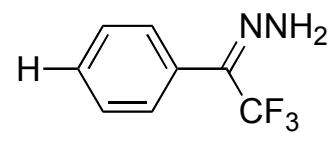

12

1-(2,2,2-Trifluoro-1-phenylethylidene)hydrazine (12):

Reaction was performed using $0.17 \mathrm{~mL}(5.47 \mathrm{mmol})$ of hydrazine. The crude hydrazone was purified by column chromatography (silica gel, diethyl ether, $R_{f}=0.67$ ) to give $\mathbf{1 2}$ (1.023 g, 94\% yield). ${ }^{1} \mathrm{H}$ NMR (400 MHz, $\left.\mathrm{CDCl}_{3}\right) \delta$ 7.55-7.49 (m, 3H), 7.38 (d, $J=7.2$ $\mathrm{Hz}, 2 \mathrm{H}), 5.85$ (bs, $2 \mathrm{H})$. The ${ }^{1} \mathrm{H}$ NMR data were consistent with the published values ${ }^{1}$. 


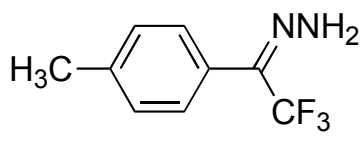

$13 a$

\section{1-(2,2,2-Trifluoro-1-p-tolylethylidene)hydrazine (13a):}

The reaction was performed using $0.080 \mathrm{~mL}(2.53 \mathrm{mmol})$ of hydrazine. The crude hydrazone was purified by column chromatography (silica gel, diethyl ether, $R_{f}=0.65$ ) to give 13a (0.496 g, 91\% yield). ${ }^{1} \mathrm{H}$ NMR $\left(400 \mathrm{MHz}, \mathrm{CDCl}_{3}\right) \delta 7.32(\mathrm{~d}, J=8 \mathrm{~Hz}, 2 \mathrm{H}), 7.26$ $(\mathrm{d}, J=8 \mathrm{~Hz}, 2 \mathrm{H}), 5.84(\mathrm{bs}, 2 \mathrm{H}), 2.41(\mathrm{~s}, 3 \mathrm{H}) ;{ }^{13} \mathrm{C} \mathrm{NMR}\left(75.45 \mathrm{MHz}, \mathrm{CDCl}_{3}\right) \delta 140.5$, $135.6\left(\mathrm{q}, J_{C C F}=34.4 \mathrm{~Hz}\right), 130.2,128.5,123.8,121.3\left(\mathrm{q}, J_{C F}=272.5 \mathrm{~Hz}\right), 21.3 ;{ }^{19} \mathrm{~F} \mathrm{NMR}$ (400 MHz, $\mathrm{CDCl}_{3}$ ) $\delta$-67.13; FTIR(neat): 1604, 1512, 1105, 975, 735; GCMS: 202 m/z; Anal. calcd. for C, 53.47; H, 4.49; N,13.86. Found: C, 53.23; H, 4.49; N, 13.57.

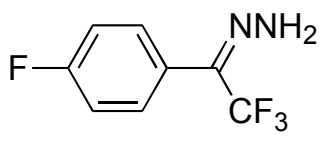

13b

\section{1-(2,2,2-Trifluoro-1-(4-fluorophenyl)ethylidene)hydrazine (13b):}

The reaction was performed using $0.08 \mathrm{~mL}(2.53 \mathrm{mmol})$ of hydrazine. The crude hydrazone was purified by column chromatography (silica gel, diethyl ether, $R_{f}=0.71$ ) to give 13b (0.472 g, 90\% yield). ${ }^{1} \mathrm{H}$ NMR (400 MHz, $\left.\mathrm{CDCl}_{3}\right) \delta 7.40-7.36(\mathrm{~m}, 2 \mathrm{H}), 7.26-$ $7.20(\mathrm{~m}, 2 \mathrm{H}), 5.85(\mathrm{bs}, 2 \mathrm{H}) ;{ }^{13} \mathrm{C} \mathrm{NMR}\left(75.45 \mathrm{MHz}, \mathrm{CDCl}_{3}\right) \delta 163.6\left(\mathrm{~d}, J_{C F}=250.8 \mathrm{~Hz}\right)$, $134.2\left(\mathrm{q}, J_{C C F}=34.4 \mathrm{~Hz}\right), 131.0\left(\mathrm{~d}, J_{C C C F}=8.6 \mathrm{~Hz}\right), 122.7\left(\mathrm{~d}, J_{C C C F}=3.4 \mathrm{~Hz}\right), 121.1(\mathrm{q}$, $\left.J_{C F}=272.0 \mathrm{~Hz}\right), 116.8\left(\mathrm{~d}, J_{C C F}=21.7 \mathrm{~Hz}\right) ;{ }^{19} \mathrm{~F} \mathrm{NMR}\left(400 \mathrm{MHz}, \mathrm{CDCl}_{3}\right) \delta-67.20$, 109.32 (q, $J=3.0 \mathrm{~Hz}$ ); FTIR(neat): 1603, 15.09, 1109, 841; GCMS: $206 \mathrm{~m} / \mathrm{z}$; Anal. calcd. for $\mathrm{C}_{8} \mathrm{H}_{6} \mathrm{~F}_{4} \mathrm{~N}_{2}$ : C, 46.61; H, 2.93; N, 13.59. Found: C, 46.90; H, 2.95; N, 13.45.

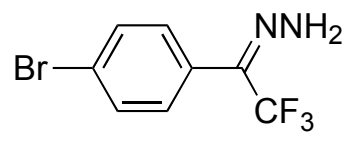

$13 \mathrm{c}$

\section{1-(1-(4-Bromophenyl)-2,2,2-trifluoroethylidene)hydrazine (13c):}

The reaction was performed using $0.250 \mathrm{~mL}(7.96 \mathrm{mmol})$ of hydrazine. The crude hydrazone was purified by column chromatography (silica gel, diethyl ether, $R_{f}=0.70$ ) to give $13 \mathrm{c}$ (1.935 g, 91\% yield with trace impurities). ${ }^{1} \mathrm{H}$ NMR (400 $\left.\mathrm{MHz}, \mathrm{CDCl}_{3}\right) \delta 7.67$ $(\mathrm{d}, J=8.4 \mathrm{~Hz}, 2 \mathrm{H}), 7.26(\mathrm{~d}, J=8.4 \mathrm{~Hz}, 2 \mathrm{H}), 5.87(\mathrm{~s}, 2 \mathrm{H}) ;{ }^{13} \mathrm{C} \mathrm{NMR}\left(75.45 \mathrm{MHz}, \mathrm{CDCl}_{3}\right)$ $\delta 134.0\left(\mathrm{q}, J_{C C F}=34.9 \mathrm{~Hz}\right), 132.9,130.4,125.6,124.8,121.0\left(\mathrm{q}, J_{C F}=272.6 \mathrm{~Hz}\right) ;{ }^{19} \mathrm{~F}$ NMR (400 MHz, $\left.\mathrm{CDCl}_{3}\right) \delta$-67.01; FTIR(neat): 1604, 1488, 1106, 827; GCMS: 265.9 $\mathrm{m} / \mathrm{z} \quad\left(\mathrm{Br}^{79} \mathrm{M}+100 \%\right), \quad 267.9 \mathrm{~m} / \mathrm{z} \quad\left(\mathrm{Br}^{81} \mathrm{M}+98.64 \%\right) ; \quad$ HRMS (EI) $\mathrm{m} / \mathrm{z}$ calcd. for $\mathrm{C}_{8} \mathrm{H}_{6} \mathrm{~F}_{3} \mathrm{~N}_{2} \mathrm{Br}: 265.9662$. Found: 265.9669 . 


\section{General Procedure for oxidation and cyclopropanation:}

The following procedure is typical for all of the diazo compound formation reactions. Trifluoromethyl hydrazone ( $1 \mathrm{mmol}$ ) was added to $\alpha, \alpha, \alpha$-trifluorotoluene (TFT, $5 \mathrm{~mL})$. The contents were degassed by bubbling argon through the solution for $5 \mathrm{~min} . \mathrm{MgSO}_{4}$ (equal mass as hydrazone) was added and then the contents were placed in an ice bath for $\sim 10$ min. At $0^{\circ} \mathrm{C}, \mathrm{MnO}_{2}(5.0 \mathrm{mmol})$ was added in one portion and the contents were allowed to stir overnight gradually warming to room temperature $(\sim 12 \mathrm{~h})$. The orange/black colored solution was then filtered through $\mathrm{SiO}_{2}$ and $\mathrm{MgSO}_{4}$ (2:1 ratio by mass) and washed with a pentane and TFT mixture (4:1 ratio by volume) until the filtrate was colorless. The orange filtrate was then degassed by bubbling argon through the solution for $5 \mathrm{~min}$ and used without further purification in the following carbenoid reaction.

The following procedure is typical for the intermolecular cyclopropanation reactions. Catalyst $(2 \mathrm{~mol} \%)$ and alkene $(5.0 \mathrm{mmol})$ were added to TFT $(5 \mathrm{~mL})$ at room temperature. A syringe pump was then used to add the diazo compound solution $(\sim 1$ mmol, described above) over $3 \mathrm{~h}$ to the catalyst solution. After the addition, the reaction was allowed to stir for $1 \mathrm{~h}$ at room temperature. The contents were then concentrated at reduced pressure and put under high vacuum. The diastereomeric excess was then determined by ${ }^{1} \mathrm{H}$ NMR on the crude residue. The crude residue was further purified by column chromatography (silica gel) to give the pure cyclopropane. The enantiomeric excess of the purified product was then determined by chiral HPLC.

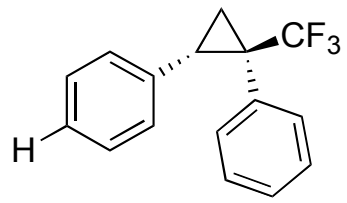

$10 \mathbf{a}$

((1R,2S)-1-(Trifluoromethylcyclopropane-1,2-diyl)dibenzene (10a):

The reaction was performed using $0.038 \mathrm{~g}(0.202 \mathrm{mmol})$ of the hydrazone 12 . The crude residue was purified by column chromatography (silica gel, pentane, $R_{f}=0.24$ ) to give 10a $\left(0.037 \mathrm{~g}, 71 \%\right.$ yield). $\mathrm{Mp}=68-69^{\circ} \mathrm{C} ;{ }^{1} \mathrm{H}$ NMR $\left(400 \mathrm{MHz}, \mathrm{CDCl}_{3}\right) \delta$ 7.20-7.08 (m, $8 \mathrm{H}), 6.77(\mathrm{dd}, J=3.6 \mathrm{~Hz}, J=6.8 \mathrm{~Hz}, 2 \mathrm{H}), 2.85(\mathrm{dd}, J=6.8 \mathrm{~Hz}, J=9.6 \mathrm{~Hz}, 1 \mathrm{H}), 1.89$ $(\mathrm{dd}, J=5.8 \mathrm{~Hz}, J=9.4 \mathrm{~Hz}, 1 \mathrm{H}), 1.70-1.67(\mathrm{~m}, 1 \mathrm{H}) ;{ }^{13} \mathrm{C} \mathrm{NMR}\left(75.45 \mathrm{MHz}, \mathrm{CDCl}_{3}\right) \delta$ 135.6, 132.5, 131.5, 128.1, 127.94, 127.88, 127.82, 126.5, 125.9 (q, $\left.J_{C F}=274.3 \mathrm{~Hz}\right), 35.8$ $\left(\mathrm{q}, J_{C C F}=32.6 \mathrm{~Hz}\right), 25.6\left(\mathrm{~d}, J_{C C C F}=2.3 \mathrm{~Hz}\right), 14.6\left(\mathrm{~d}, J_{C C C F}=2.3 \mathrm{~Hz}\right) ;{ }^{19} \mathrm{~F}$ NMR $(400$ $\left.\mathrm{MHz}, \mathrm{CDCl}_{3}\right) \delta$-69.74 ; FTIR(neat): 1498, 1320, 1281, 1135, 695, 652; GCMS: 262 m/z; HRMS (EI) $\mathrm{m} / \mathrm{z}$ calcd. for $\mathrm{C}_{16} \mathrm{H}_{13} \mathrm{~F}_{3}$ : 262.0964. Found: 262.0955; Anal. calcd. for $\mathrm{C}_{16} \mathrm{H}_{13} \mathrm{~F}_{3}$ : C, 73.27; H, 5.00. Found: $\mathrm{C}, 72.99$; H, 4.81; HPLC analysis: $>99 \%$ ee (chiralcel OJ, $1 \% i \mathrm{Pr}-\mathrm{OH}$ in hexane, $0.8 \mathrm{~mL} / \mathrm{min} \lambda=230 \mathrm{~nm}, \mathrm{t}_{\mathrm{R}}=6.42 \mathrm{~min}$, major; $\mathrm{t}_{\mathrm{R}}=7.61 \mathrm{~min}$, minor $),[\alpha]_{\mathrm{D}}^{25}=+113.0^{\circ}\left(c 1.00, \mathrm{CHCl}_{3}\right)$. 
<smiles>Cc1ccc([C@@H]2C[C@]2(c2ccccc2)C(F)(F)F)cc1</smiles>

$10 \mathrm{~b}$

1-((1R,2S)-2-(4-Methylbenzenyl)-1-(trifluoromethyl)cyclopropyl)benzene (10b): The reaction was performed using $0.011 \mathrm{~g}(0.058 \mathrm{mmole})$ of the hydrazone 12 . The crude residue was purified by column chromatography (silica gel, pentane, $R_{f}=0.24$ ) to give $10 b(0.012 \mathrm{~g}, 72 \%$ yield $) . \mathrm{Mp}=70-71{ }^{\circ} \mathrm{C} ;{ }^{1} \mathrm{H}$ NMR $\left(400 \mathrm{MHz}, \mathrm{CDCl}_{3}\right) \delta$ 7.24-7.14 (m, $5 \mathrm{H}), 6.90(\mathrm{~d}, J=8.0 \mathrm{~Hz}, 2 \mathrm{H}), 6.66(\mathrm{~d}, J=8.0 \mathrm{~Hz}, 2 \mathrm{H}), 2.81(\mathrm{dd}, J=7.0 \mathrm{~Hz}, J=9.4 \mathrm{~Hz}$, $1 \mathrm{H}), 2.23(\mathrm{~s}, 3 \mathrm{H}) 1.87(\mathrm{dd}, J=5.8 \mathrm{~Hz}, J=9.4 \mathrm{~Hz}, 1 \mathrm{H}), 1.66-1.63(\mathrm{~m}, 1 \mathrm{H}) ;{ }^{13} \mathrm{C} \mathrm{NMR}$ $\left(75.45 \mathrm{MHz}, \mathrm{CDCl}_{3}\right) \delta 136.0,132.5,131.7,128.5,128.1,127.9,127.8,124.1,35.6$ (q, $\left.J_{C C F}=32.6 \mathrm{~Hz}\right), 25.3\left(\mathrm{~d}, J_{C C C F}=2.3 \mathrm{~Hz}\right), 20.9,14.7 ;{ }^{19} \mathrm{~F} \mathrm{NMR}\left(400 \mathrm{MHz}, \mathrm{CDCl}_{3}\right) \delta$ 69.73; FTIR(neat): 1519, 1380, 1133, 699, 652; GCMS: $276 \mathrm{~m} / \mathrm{z}$; Anal. calcd. for $\mathrm{C}_{17} \mathrm{H}_{15} \mathrm{~F}_{3}$ : C, 73.90; H, 5.47. Found: $\mathrm{C}, 73.62 ; \mathrm{H}, 5.47$; HPLC analysis: $90 \%$ ee (chiralcel $\mathrm{OJ}, 1 \% i \mathrm{Pr}-\mathrm{OH}$ in hexane, $1.0 \mathrm{~mL} / \mathrm{min} \lambda=230 \mathrm{~nm}, \mathrm{t}_{\mathrm{R}}=4.9 \mathrm{~min}$, major; $\mathrm{t}_{\mathrm{R}}=6.1 \mathrm{~min}$, minor $),[\alpha]_{\mathrm{D}}{ }^{25}=+57.2\left(c 0.50, \mathrm{CHCl}_{3}\right)$

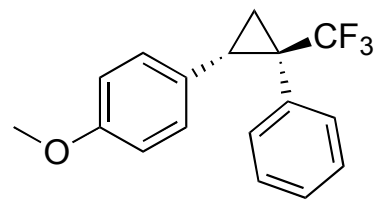

10c

1-((1R,2S)-2(4-Methoxybenzenyl)-1-(trifluoromethyl)cyclopropyl)benzene (10c):

The reaction was performed using $0.039 \mathrm{~g}(0.207 \mathrm{mmol})$ of the hydrazone $\mathbf{1 2}$. The crude residue was purified by column chromatography (silica gel, diethyl ether / pentane 1:25 ratio by volume, $\left.R_{f}=0.50\right)$ to give $10 \mathrm{c}(0.047 \mathrm{~g}, 78 \%$ yield $) .{ }^{1} \mathrm{H} \mathrm{NMR}\left(400 \mathrm{MHz}, \mathrm{CDCl}_{3}\right)$ $7.12-7.13(\mathrm{~m}, 5 \mathrm{H}), 6.70-6.62(\mathrm{~m}, 4 \mathrm{H}), 3.71(\mathrm{~s}, 3 \mathrm{H}), 2.80(\mathrm{dd}, J=7.2 \mathrm{~Hz}, J=9.6 \mathrm{~Hz}, 1 \mathrm{H})$, $1.86(\mathrm{dd}, J=5.6 \mathrm{~Hz}, J=9.6 \mathrm{~Hz}, 1 \mathrm{H}), 1.62-1.59(\mathrm{~m}, 1 \mathrm{H}) ;{ }^{13} \mathrm{C} \mathrm{NMR}\left(75.45 \mathrm{MHz}, \mathrm{CDCl}_{3}\right)$ $\delta 158.2,132.5,131.7,128.8,128.0127 .9,127.5,125.9$ (q, $\left.J_{C F}=274.3 \mathrm{~Hz}\right), 113.3,55.0$, $35.3\left(\mathrm{q}, J_{C C F}=32.7 \mathrm{~Hz}\right), 25.0\left(\mathrm{~d}, J_{C C C F}=2.9 \mathrm{~Hz}\right), 14.6\left(\mathrm{~d}, J_{C C C F}=2.3 \mathrm{~Hz}\right) ;{ }^{19} \mathrm{~F}$ NMR $(400$ $\left.\mathrm{MHz}, \mathrm{CDCl}_{3}\right) \delta$-69.63; FTIR(neat): 3032, 2810, 1517, 1131, 699, 652; GCMS: 292 m/z; Anal. calcd. for $\mathrm{C}_{17} \mathrm{H}_{15} \mathrm{~F}_{3} \mathrm{O}$ : C, 69.85; H, 5.17. Found: $\mathrm{C}, 69.74 ; \mathrm{H}, 5.03$; HPLC analysis: $88 \%$ ee (chiralcel OJ, $1 \% i \operatorname{Pr}-\mathrm{OH}$ in hexane, $0.8 \mathrm{~mL} / \mathrm{min} \lambda=230 \mathrm{~nm}, \mathrm{t}_{\mathrm{R}}=8.6 \mathrm{~min}$, major; $\mathrm{t}_{\mathrm{R}}=11.4$ min, minor $),[\alpha]_{\mathrm{D}}{ }^{25}=+105.4\left(c 4.50, \mathrm{CHCl}_{3}\right)$.<smiles>FC(F)(F)C1(c2ccccc2)C[C@H]1c1ccc(Cl)cc1</smiles>

10d

1-((1R,2S)-2-(4-Chlorobenzenyl)-1-(trifluoromethyl)cyclopropyl)benzene (10d):

The reaction was performed using $0.011 \mathrm{~g}(0.058 \mathrm{mmol})$ of the hydrazone 12 . The crude residue was purified by column chromatography (silica gel, pentane, $R_{f}=0.26$ ) to give 
10d $\left(0.011 \mathrm{~g}, 64 \%\right.$ yield). ${ }^{1} \mathrm{H}$ NMR $\left(400 \mathrm{MHz}, \mathrm{CDCl}_{3}\right) \delta$ 7.23-7.11 (m, 5H), 7.07-7.05 (m, 2H), 6.70-6.68 (m, 2H), 2.81 (dd, $J=7.2 \mathrm{~Hz}, J=9.6 \mathrm{~Hz}, 1 \mathrm{H}), 1.91$ (dd, $J=6.2 \mathrm{~Hz}, J$ $=9.8 \mathrm{~Hz}, 1 \mathrm{H}), 1.66-1.63(\mathrm{~m}, 1 \mathrm{H}) ;{ }^{13} \mathrm{C}$ NMR $\left(75.35 \mathrm{MHz}, \mathrm{CDCl}_{3}\right) \delta 134.3,132.4,132.3$, $131.1,129.1,128.3,128.1,128.0,125.7\left(\mathrm{q}, J_{C F}=274.3 \mathrm{~Hz}\right), 35.9\left(\mathrm{q}, J_{C C F}=32.7 \mathrm{~Hz}\right)$, $25.0\left(\mathrm{~d}, J_{C C C F}=2.3 \mathrm{~Hz}\right), 14.8 ;{ }^{19} \mathrm{~F}$ NMR $\left(400 \mathrm{MHz}, \mathrm{CDCl}_{3}\right) \delta-69.84$; FTIR(neat): 3032 , 1497, 1134, 699, 653; GCMS: $296 \mathrm{~m} / \mathrm{z}$; HRMS (ES) m/z calcd. for $\mathrm{C}_{16} \mathrm{H}_{12} \mathrm{ClF}_{3}$ : 296.0574. Found: 296.0574; HPLC analysis: $90 \%$ ee (chiralcel OJ, $1 \% i \mathrm{Pr}-\mathrm{OH}$ in hexane, $1.0 \mathrm{~mL} / \min \lambda=230 \mathrm{~nm}, \mathrm{t}_{\mathrm{R}}=6.1 \mathrm{~min}$, major; $\mathrm{t}_{\mathrm{R}}=8.4 \mathrm{~min}$, minor $),[\alpha]_{\mathrm{D}}{ }^{25}=+55.5(\mathrm{c}$ $\left.0.80, \mathrm{CHCl}_{3}\right)$.

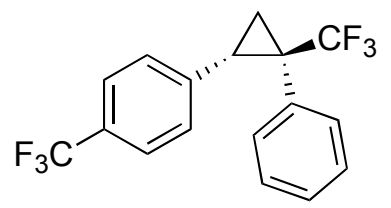

$10 \mathrm{e}$

1-((1R,2S)-2-(4-(Trifluoromethyl)phenyl)-1-(trifluoromethyl)cyclopropyl)benzene (10e):

The reaction was performed using $0.025 \mathrm{~g}(0.133 \mathrm{mmol})$ of the hydrazone 12 . The crude residue was purified by column chromatography (silica gel, pentane, $\mathrm{R}_{\mathrm{f}}=0.45$ ) to give 10e (0.027 g, 61\% yield). ${ }^{1} \mathrm{H}$ NMR (400 MHz, $\left.\mathrm{CDCl}_{3}\right) \delta 7.36(\mathrm{~d}, J=8.0 \mathrm{~Hz}, 2 \mathrm{H}), 7.25-7.11$ $(\mathrm{m}, 5 \mathrm{H}), 6.86(\mathrm{~d}, J=8.0 \mathrm{~Hz}, 2 \mathrm{H}), 2.88(\mathrm{dd}, J=7.2 \mathrm{~Hz}, J=9.2 \mathrm{~Hz}, 1 \mathrm{H}), 1.97$ (dd, $J=6.0$ $\mathrm{Hz}, 1 \mathrm{H}), 1.74-1.71(\mathrm{~m}, 1 \mathrm{H}) ;{ }^{13} \mathrm{C} \operatorname{NMR}\left(75.45 \mathrm{MHz}, \mathrm{CDCl}_{3}\right) \delta 140.1,132.4,130.8$, 128.4,128.2, 128.1, 125.7 (q, $\left.J_{C F}=274.3 \mathrm{~Hz}\right), 124.7$ (q, $\left.J_{C C F}=4.0 \mathrm{~Hz}\right), 124.0$ (q, $J_{C F}=280$ $\mathrm{Hz}), 36.4\left(\mathrm{q}, J_{C C F}=32.7 \mathrm{~Hz}\right), 25.3\left(\mathrm{~d}, J_{C C C F}=2.3 \mathrm{~Hz}\right), 15.2 ;{ }^{19} \mathrm{~F}$ NMR $\left(400 \mathrm{MHz}, \mathrm{CDCl}_{3}\right)$ $\delta$-62.49, -70.05; FTIR(neat): 3032, 1620, 1323, 1070, 700, 655; GCMS: 330 m/z; HRMS (EI) $\mathrm{m} / \mathrm{z}$ calcd. for $\mathrm{C}_{17} \mathrm{H}_{12} \mathrm{~F}_{6}$ : 330.0839. Found: 330.0840; HPLC analysis: $>94 \%$ ee (chiralcel OJ, $0 \% i \mathrm{Pr}-\mathrm{OH}$ in hexane, $1.0 \mathrm{~mL} / \mathrm{min} \lambda=230 \mathrm{~nm}, \mathrm{t}_{\mathrm{R}}=10.9 \mathrm{~min}$, major; $\mathrm{t}_{\mathrm{R}}=$ 16.5 min, minor), $[\alpha]_{\mathrm{D}}{ }^{25}=+109.1\left(c 1.10, \mathrm{CHCl}_{3}\right)$.

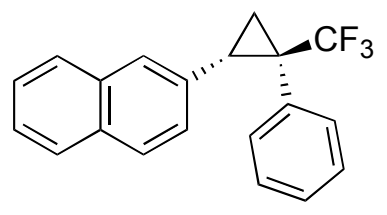

$10 f$

1-((1R,2S)-2-Naphthyl-1-(trifluoromethyl)cyclopropyl)benzene (10f):

The reaction was performed using $0.024 \mathrm{~g}(0.128 \mathrm{mmol})$ of the hydrazone 12 . The crude residue was purified by column chromatography (silica gel, pentane, $R_{f}=0.19$ ) to give $\mathbf{5 f}$ (0.028 g, 75\% yield). $\mathrm{Mp}=81-82^{\circ} \mathrm{C} ;{ }^{1} \mathrm{H}$ NMR $\left(400 \mathrm{MHz}, \mathrm{CDCl}_{3}\right) \delta 7.70-7.68(\mathrm{~m}, 1 \mathrm{H})$, 7.61-7.59 (m, 1H), $7.53(\mathrm{~d}, J=8.4 \mathrm{~Hz}, 1 \mathrm{H}), 7.40-7.34(\mathrm{~m}, 2 \mathrm{H}), 7.31(\mathrm{~s}, 1 \mathrm{H}), 7.18-7.08$ $(\mathrm{m}, 5 \mathrm{H}), 6.81(\mathrm{dd}, J=1.6 \mathrm{~Hz}, J=8.4 \mathrm{~Hz}, 1 \mathrm{H}), 2.99(\mathrm{dd}, J=7.2 \mathrm{~Hz}, J=9.6 \mathrm{~Hz}, 1 \mathrm{H}), 1.95$ $(\mathrm{dd}, J=6.4 \mathrm{~Hz}, J=9.6 \mathrm{~Hz}, 1 \mathrm{H}), 1.82-1.78(\mathrm{~m}, 1 \mathrm{H}) ;{ }^{13} \mathrm{C} \mathrm{NMR}\left(75.45 \mathrm{MHz}, \mathrm{CDCl}_{3}\right) \delta$ $133.3,133.0,132.1,131.4,132.4,128.2,128.0,127.5,127.4,127.3,126.9,126.0,125.91$ $\left(\mathrm{q}, J_{C F}=274.3 \mathrm{~Hz}\right), 125.90,125.5,35.9\left(\mathrm{q}, J_{C C F}=32.1 \mathrm{~Hz}\right), 25.8\left(\mathrm{q}, J_{C C C F}=2.3 \mathrm{~Hz}\right)$, $14.9\left(\mathrm{q}, J_{C C C F}=2.3 \mathrm{~Hz}\right) ;{ }^{19} \mathrm{~F}$ NMR $\left(400 \mathrm{MHz}, \mathrm{CDCl}_{3}\right) \delta-69.71$; FTIR(neat): 3059, 1601, 1347, 1294, 1135, 1126, 699; GCMS: $312 \mathrm{~m} / \mathrm{z}$; Anal. calcd. for $\mathrm{C}_{20} \mathrm{H}_{15} \mathrm{~F}_{3}: \mathrm{C}, 76.91 ; \mathrm{H}$, 
4.84. Found: C, 76.55; H, 4.72; HPLC analysis: $89 \%$ ee (chiralcel OJ, $1 \% i \mathrm{Pr}-\mathrm{OH}$ in hexane, $0.8 \mathrm{~mL} / \min \lambda=230 \mathrm{~nm}, \mathrm{t}_{\mathrm{R}}=7.9 \mathrm{~min}$, major; $\mathrm{t}_{\mathrm{R}}=15.3 \mathrm{~min}$, minor $),[\alpha]_{\mathrm{D}}{ }^{25}=+84.6$ (c $2.30, \mathrm{CHCl}_{3}$ ).

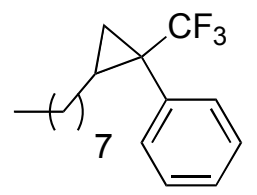

$10 \mathrm{~g}$

1-(1-(Trifluoromethyl)-2-octylcyclopropyl)benzene (10g):

To a flame dried round bottom flask equipped with stir bar under argon, 1-decene (5 mmole, $0.200 \mathrm{~g}, 5$ eq.), TFT $(10 \mathrm{~mL})$, and catalyst $\mathrm{Rh}_{2}(\mathrm{OAc})_{4}(2 \mathrm{~mol} \%, 9 \mathrm{mg})$ were added and degassed by argon. A syringe pump was then used to add diazo compound 9 (1 mmole, $0.186 \mathrm{~g}$ in TFT and pentane $1: 1$ by volume, $10 \mathrm{~mL}$ ) over $3 \mathrm{hr}$ to the catalyst solution. After the addition, reaction was allowed to stir for $1 \mathrm{~h}$. Contents were concentrated in vacuo and then put under high vacuum. This residue was further purified by chromatography (silica gel, pentane, $\left.R_{f}=0.61\right)$ to give $10 \mathrm{~g}\left(0.104 \mathrm{~g}, 20 \%\right.$ yield). ${ }^{1} \mathrm{H}$ NMR (400 MHz, $\left.\mathrm{CDCl}_{3}\right) \delta$ 7.40-7.30 (m, 5H), 1.55-1.21 (m, 15H), 0.88-0.85 (m, 4H), 0.53-0.49 (m, 1H); ${ }^{13} \mathrm{C}$ NMR $\left(125.7 \mathrm{MHz}, \mathrm{CDCl}_{3}\right)(\delta$ 77.00ppm) $\delta 133.2,131.9,128.2$, $128.1,126.4\left(\mathrm{q}, J_{C F}=273.5 \mathrm{~Hz}\right), 32.3\left(\mathrm{q}, J_{C C F}=32.3 \mathrm{~Hz}\right), 31.8,29.9,29.5,29.3,29.2$, 29.1, 22.6, 20.9 (q, $\left.J_{C C C F}=2.3 \mathrm{~Hz}\right), 15.0\left(\mathrm{q}, J_{C C C F}=2.8 \mathrm{~Hz}\right), 14.1 ;{ }^{19} \mathrm{~F}$ NMR (400 MHz, $\left.\mathrm{CDCl}_{3}\right) \delta$-71.89; FTIR(neat): 2927, 2856, 1303, 1151, 1134, 700, 659; GCMS: 298 m/z; Anal. calcd. for $\mathrm{C}_{18} \mathrm{H}_{25} \mathrm{~F}_{3}$ : C, 72.45; H, 8.44. Found: C, 72.57; H, 8.46. Diastereomic Excess and Enantiomeric Excess were not determined.

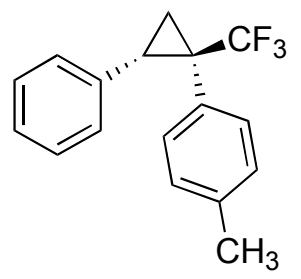

$14 \mathbf{a}$

1-Methyl-4-((1R,2S)-2-phenyl-1-(trifluoromethyl)cyclopropyl)benzene (14a):

The reaction was performed using $0.023 \mathrm{~g}(0.114 \mathrm{mmol})$ of the hydrazone 13a. The crude residue was purified by column chromatography (silica gel, pentane, $R_{f}=0.23$ ) to give 14a $(0.023 \mathrm{~g}, 75 \%$ yield $) . \mathrm{Mp}=67-68^{\circ} \mathrm{C} ;{ }^{1} \mathrm{H}$ NMR $\left(400 \mathrm{MHz}, \mathrm{CDCl}_{3}\right) \delta 7.10-7.08$ (m, $3 \mathrm{H}), 7.02(\mathrm{~d}, J=8 \mathrm{~Hz}, 2 \mathrm{H}), 6.96(\mathrm{~d}, J=8.4 \mathrm{~Hz}, 2 \mathrm{H}), 6.80-6.78(\mathrm{~m}, 2 \mathrm{H}), 2.81(\mathrm{dd}, J=7.2$ $\mathrm{Hz}, J=9.6 \mathrm{~Hz}, 1 \mathrm{H}), 2.25$ (s, 3H), $1.85(\mathrm{dd}, J=5.8 \mathrm{~Hz}, J=9.4 \mathrm{~Hz}, 1 \mathrm{H}), 1.65-1.63$ (m, $1 \mathrm{H}) ;{ }^{13} \mathrm{C}$ NMR $\left(75.45 \mathrm{MHz}, \mathrm{CDCl}_{3}\right) \delta 137.9,135.8,132.3,128.7,128.4,128.0,127.8$, $126.0\left(\mathrm{q}, J_{C F}=273.7 \mathrm{~Hz}\right), 126.4,35.4\left(\mathrm{q}, J_{C C F}=32.6 \mathrm{~Hz}\right), 25.6\left(\mathrm{~d}, J_{C C C F}=2.3 \mathrm{~Hz}\right), 21.1$, $14.7\left(\mathrm{~d}, J_{C C C F}=2.3 \mathrm{~Hz}\right) ;{ }^{19} \mathrm{~F}$ NMR $\left(400 \mathrm{MHz}, \mathrm{CDCl}_{3}\right) \delta$-69.78; FTIR(neat): 3031, 1518, 1133, 910, 811, 695; GCMS: $276 \mathrm{~m} / \mathrm{z}$; Anal. calcd. for $\mathrm{C}_{17} \mathrm{H}_{15} \mathrm{~F}_{3}$ : C, 73.90; H, 5.47. Found: C, 74.00; H, 5.54; HPLC analysis: $99 \%$ ee (chiralcel OJ, $0.5 \% \mathrm{Pr}-\mathrm{OH}$ in hexane, $1.0 \mathrm{~mL} / \mathrm{min} \lambda=230 \mathrm{~nm}, \mathrm{t}_{\mathrm{R}}=4.5 \mathrm{~min}$, major; $\mathrm{t}_{\mathrm{R}}=5.7 \mathrm{~min}$, minor), $[\alpha]_{\mathrm{D}}{ }^{25}=+124.4$ (c $1.00, \mathrm{CHCl}_{3}$ ). 


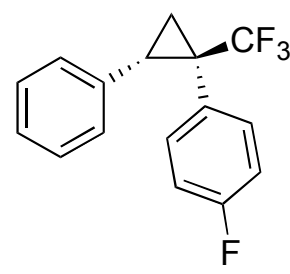

14b

1-Fluoro-4-((1R,2S)-2-phenyl-1-(trifluoromethyl)cyclopropyl)benzene (14b):

The reaction was performed using $0.024 \mathrm{~g}(0.116 \mathrm{mmol})$ of the hydrazone $13 \mathrm{~b}$. The crude residue was purified by column chromatography (silica gel, pentane, $R_{f}=0.30$ ) to give 14b (0.026 g, 78\% yield). ${ }^{1} \mathrm{H}$ NMR (400 MHz, $\left.\mathrm{CDCl}_{3}\right) \delta 7.11-7.08(\mathrm{~m}, 5 \mathrm{H}), 6.85$ (m, 2H), $6.76(\mathrm{dd}, J=3.2 \mathrm{~Hz}, J=6.8 \mathrm{~Hz}, 2 \mathrm{H}) 2.84(\mathrm{dd}, J=6.8 \mathrm{~Hz}, J=9.6 \mathrm{~Hz}, 1 \mathrm{H}), 1.88$ (dd, $J=5.8 \mathrm{~Hz}, J=9.4 \mathrm{~Hz}, 1 \mathrm{H}), 1.65(\mathrm{~m}, 1 \mathrm{H}) ;{ }^{13} \mathrm{C} \mathrm{NMR}\left(75.45 \mathrm{MHz}, \mathrm{CDCl}_{3}\right) \delta 162.5\left(\mathrm{~d}, J_{C F}=\right.$ $247.9 \mathrm{~Hz}), 135.3,134.3,134.2,128.0,127.9,126.6,125.8\left(\mathrm{q}, J_{C F}=274.8 \mathrm{~Hz}\right), 115.0(\mathrm{~d}$, $\left.J_{C C F}=21.8 \mathrm{~Hz}\right), 35.0\left(\mathrm{q}, J_{C C F}=32.6 \mathrm{~Hz}\right), 25.7\left(\mathrm{~d}, J_{C C C F}=2.3 \mathrm{~Hz}\right), 14.6\left(\mathrm{~d}, J_{C C C F}=2.3\right.$ $\mathrm{Hz}$ ); ${ }^{19} \mathrm{~F}$ NMR (400 MHz, $\mathrm{CDCl}_{3}$ ) $\delta$-69.82, -113.09; FTIR(neat): 2918, 1513, 1302, 1133, 909, 728; GCMS: 280 m/z; Anal. calcd. for $\mathrm{C}_{16} \mathrm{H}_{12} \mathrm{~F}_{4}: \mathrm{C}, 68.57$; H, 4.31. Found: $\mathrm{C}$, 68.64; H, 4.33; HPLC analysis: $97 \%$ ee (chiralcel OJ, $1.0 \% i \mathrm{Pr}-\mathrm{OH}$ in hexane, 1.0 $\mathrm{mL} / \mathrm{min} \lambda=230 \mathrm{~nm}, \mathrm{t}_{\mathrm{R}}=4.8 \mathrm{~min}$, major; $\mathrm{t}_{\mathrm{R}}=7.4 \mathrm{~min}$, minor $),[\alpha]_{\mathrm{D}}{ }^{25}=+99.8$ (c 1.05, $\left.\mathrm{CHCl}_{3}\right)$.

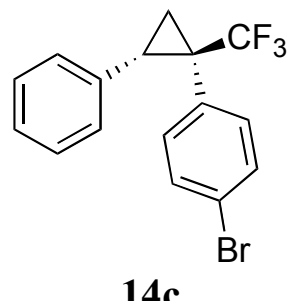

\section{1-Bromo-4-((1R,2S)-2-phenyl-1-(trifluoromethyl)cyclopropyl)benzene (14c):}

The reaction was performed using $0.105 \mathrm{~g}(0.393 \mathrm{mmol})$ of the hydrazone $13 \mathrm{c}$. The crude residue was purified by column chromatography (silica gel, pentane, $R_{f}=0.26$ ) to give 14c (0.103 g, 77\% yield). ${ }^{1} \mathrm{H}$ NMR (400 MHz, $\left.\mathrm{CDCl}_{3}\right) \delta 7.29$ (d, $\left.J=8.4 \mathrm{~Hz}, 2 \mathrm{H}\right), 7.12-$ $7.10(\mathrm{~m}, 3 \mathrm{H}), 7.00(\mathrm{~d}, J=8.4 \mathrm{~Hz}, 2 \mathrm{H}), 6.79-6.77(\mathrm{~m}, 2 \mathrm{H}), 2.85(\mathrm{dd}, J=7.2 \mathrm{~Hz}, J=9.6$ $\mathrm{Hz}, 1 \mathrm{H}), 1.87(\mathrm{dd}, J=6.0 \mathrm{~Hz}, J=9.6 \mathrm{~Hz}, 1 \mathrm{H}), 1.66-1.63(\mathrm{~m}, 1 \mathrm{H}) ;{ }^{13} \mathrm{C} \mathrm{NMR}(75.45 \mathrm{MHz}$, $\left.\mathrm{CDCl}_{3}\right) \delta 135.0,134.1,131.2,130.7,128.0,127.9,126.7,125.6\left(\mathrm{q}, J_{C F}=274.2 \mathrm{~Hz}\right)$, $122.6,35.2\left(\mathrm{q}, J_{C C F}=32.7 \mathrm{~Hz}\right), 25.6\left(\mathrm{~d}, J_{C C C F}=2.3 \mathrm{~Hz}\right), 14.5\left(\mathrm{~d}, J_{C C C F}=2.3 \mathrm{~Hz}\right) ;{ }^{19} \mathrm{~F}$ NMR (400 MHz, $\mathrm{CDCl}_{3}$ ) $\delta$-69.71; FTIR(neat): 3033, 1396, 1133, 975, 696; GCMS: 340 $\mathrm{m} / \mathrm{z}, 342 \mathrm{~m} / \mathrm{z}$; Anal. calcd. for $\mathrm{C}_{16} \mathrm{H}_{12} \mathrm{BrF}_{3}$ : C, 56.33; H, 3.55. Found: C, 56.34; $\mathrm{H}, 3.45$; HPLC analysis: $98 \%$ ee (chiralcel OJ, $1.0 \% i \mathrm{Pr}-\mathrm{OH}$ in hexane, $1.0 \mathrm{~mL} / \mathrm{min} \lambda=230 \mathrm{~nm}$, $\mathrm{t}_{\mathrm{R}}=4.8$ min, major; $\mathrm{t}_{\mathrm{R}}=6.5 \mathrm{~min}$, minor $),[\alpha]_{\mathrm{D}}{ }^{25}=+123.8\left(\right.$ c 2.30, $\left.\mathrm{CHCl}_{3}\right)$. 


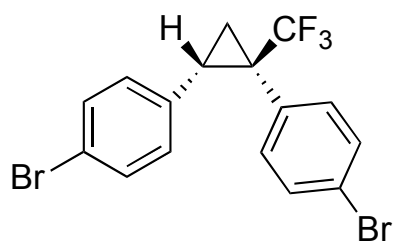

15a

4,4'-(1R,2S)-1-(Trifluoromethyl)cyclopropane-1,2-diyl)bis(bromobenzene) (15a):

The reaction was performed using $0.029 \mathrm{~g}(0.109 \mathrm{mmol})$ of the hydrazone $13 \mathrm{c}$. The crude residue was purified by column chromatography ( silica gel, pentane, $R_{f}=0.32$ ) to give 15a (0.036 g, $78 \%$ yield). ${ }^{1} \mathrm{H}$ NMR $\left(400 \mathrm{MHz}, \mathrm{CDCl}_{3}\right) \delta 7.29$ (d, $\left.J=8.4 \mathrm{~Hz}, 2 \mathrm{H}\right), 7.13-$ $7.11(\mathrm{~m}, 2 \mathrm{H}), 7.00(\mathrm{~d}, J=8.4 \mathrm{~Hz}, 2 \mathrm{H}), 6.80-6.77(\mathrm{~m}, 2 \mathrm{H}), 2.85$ (dd, $J=7.2 \mathrm{~Hz}, J=9.6$ $\mathrm{Hz}, 1 \mathrm{H}), 1.88(\mathrm{dd}, J=6.0 \mathrm{~Hz}, 9.6 \mathrm{~Hz}, 1 \mathrm{H}), 1.70-1.63(\mathrm{~m}, 1 \mathrm{H}) ;{ }^{13} \mathrm{C}$ NMR $(75.45 \mathrm{MHz}$, $\left.\mathrm{CDCl}_{3}\right) \delta 134.3,134.0,131.5,131.2,130.2,129.5,125.4\left(\mathrm{q}, J_{C F}=274.9 \mathrm{~Hz}\right), 122.9$, 120.7, $35.4\left(\mathrm{q}, J_{C C F}=32.7 \mathrm{~Hz}\right), 25.1,14.8 ;{ }^{19} \mathrm{~F} \mathrm{NMR}\left(400 \mathrm{MHz}, \mathrm{CDCl}_{3}\right) \delta$-69.86; FTIR(neat): 1593, 1491, 1292, 1137, 1011, 724, 668; HRMS (EI) m/z calcd. for $\mathrm{C}_{16} \mathrm{H}_{11} \mathrm{~F}_{3} \mathrm{Br}_{2}$ : 417.9174. Found: 417.9175; HPLC analysis: $>99 \%$ ee (chiralcel OJ, $1.0 \%$ $i \mathrm{Pr}-\mathrm{OH}$ in hexane, $1.0 \mathrm{~mL} / \mathrm{min} \lambda=230 \mathrm{~nm}, \mathrm{t}_{\mathrm{R}}=7.3 \mathrm{~min}$, major; $\mathrm{t}_{\mathrm{R}}=11.7 \mathrm{~min}$, minor), $[\alpha]_{\mathrm{D}}^{25}=+117.0\left(c 8.10, \mathrm{CHCl}_{3}\right)$.<smiles>O=[N+]([O-])c1ccc([C@H]2C[C@]2(c2ccc(Br)cc2)C(F)(F)F)cc1</smiles>

$15 b$

1-Bromo-4-((1R,2S)-2-(4-nitrophenyl)-1-(trifluoromethyl)cyclopropyl)benzene (15b):

The reaction was performed using $0.150 \mathrm{~g}(0.562 \mathrm{mmol})$ of the hydrazone $13 \mathrm{c}$. The crude residue was purified by column chromatography (silica gel, 20:1 v/v pentane / diethyl ether then $2: 1 \mathrm{v} / \mathrm{v}$ pentane / diethyl ether) to give $\mathbf{1 5 b}(0.162 \mathrm{~g}, 75 \%$ yield $) .{ }^{1} \mathrm{H}$ NMR $\left(400 \mathrm{MHz}, \mathrm{CDCl}_{3}\right) \delta 8.00(\mathrm{~d}, J=8.4 \mathrm{~Hz}, 2 \mathrm{H}), 7.34(\mathrm{~d}, J=8.4 \mathrm{~Hz}, 2 \mathrm{H}), 6.99$ (d, $J=8.4$ $\mathrm{Hz}, 2 \mathrm{H}), 6.93(\mathrm{~d}, 8.4 \mathrm{~Hz}, 2 \mathrm{H}), 2.94(\mathrm{dd}, J=6.8 \mathrm{~Hz}, J=9.2 \mathrm{~Hz}, 1 \mathrm{H}), 2.03$ (dd, $J=6.4 \mathrm{~Hz}$, $J=9.6 \mathrm{~Hz}, 1 \mathrm{H}), 1.77-1.74(\mathrm{~m}, 1 \mathrm{H}){ }^{13} \mathrm{C} \mathrm{NMR}\left(75.45 \mathrm{MHz}, \mathrm{CDCl}_{3}\right) \delta 146.7,143.2,133.7$, $132.5,131.9,131.7,129.6,128.5,125.2\left(\mathrm{q}, J_{C F}=257.7 \mathrm{~Hz}\right), 123.2,36.4\left(\mathrm{q}, J_{C C F}=33.2\right.$ $\mathrm{Hz}$ ), 25.4, 15.5; ${ }^{19} \mathrm{~F}$ NMR (400 MHz, $\left.\mathrm{CDCl}_{3}\right) \delta$-70.14; FTIR(neat): 1601, 1518,1343, 1135, 818, 718, 699; Anal. calcd. for $\mathrm{C}_{16} \mathrm{H}_{11} \mathrm{BrF}_{3} \mathrm{NO}_{2}$ : C, 49.76; H, 2.87; N, 3.63. Found: $\mathrm{C}, 49.83 ; \mathrm{H}, 2.87 ; \mathrm{N}, 3.61$; HPLC analysis: $>99 \%$ ee (chiralcel OJ, $2.5 \% \mathrm{iPr}-\mathrm{OH}$ in hexane, $0.8 \mathrm{~mL} / \mathrm{min} \lambda=230 \mathrm{~nm}, \mathrm{t}_{\mathrm{R}}=18.4 \mathrm{~min}$, major; $\mathrm{t}_{\mathrm{R}}=23.8 \mathrm{~min}$, minor $),[\alpha]_{\mathrm{D}}{ }^{25}=+172.7$ (c $\left.0.30, \mathrm{CHCl}_{3}\right)$. 


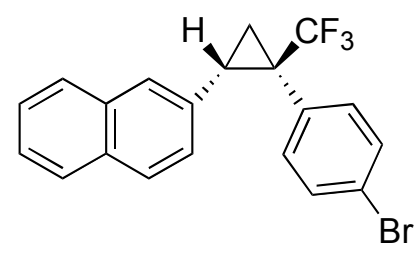

$15 \mathrm{c}$

1-Bromo-4-((1R,2S)-2-naphthyl-1-(trifluoromethyl)cyclopropyl)benzene (15c): The reaction was performed using $0.034 \mathrm{~g}(0.127 \mathrm{mmol})$ of the hydrazone 13c. The crude residue was purified by column chromatography (silica gel, pentane, $R_{f}=0.21$ ) to give 15c $\left(0.040 \mathrm{~g}, 80 \%\right.$ yield). ${ }^{1} \mathrm{H}$ NMR $\left(400 \mathrm{MHz}, \mathrm{CDCl}_{3}\right) \delta$ 7.73-7.71 (m, $\left.1 \mathrm{H}\right), 7.65-7.63$ (m, $1 \mathrm{H}), 7.58(\mathrm{~d}, J=8.4 \mathrm{~Hz}, 1 \mathrm{H}), 7.43-7.35(\mathrm{~m}, 3 \mathrm{H}), 7.25-7.23(\mathrm{~m}, 2 \mathrm{H}), 7.03(\mathrm{~d}, J=8.0$ $\mathrm{Hz}, 2 \mathrm{H}), 6.83(\mathrm{~d}, J=8.8 \mathrm{~Hz}, 1 \mathrm{H}), 3.01(\mathrm{dd}, J=7.2 \mathrm{~Hz}, J=9.2 \mathrm{~Hz}, 1 \mathrm{H}), 1.96(\mathrm{dd}, J=5.6$ $\mathrm{Hz}, J=9.6 \mathrm{~Hz}, 1 \mathrm{H}), 1.80-1.77(\mathrm{~m}, 1 \mathrm{H}) ;{ }^{13} \mathrm{C} \mathrm{NMR}\left(75.45 \mathrm{MHz}, \mathrm{CDCl}_{3}\right) \delta$ 134.0, 133.0, $132.7,132.2,131.3,130.6,127.6,127.55,127.47,127.40,126.2,125.7,125.6\left(\mathrm{q}, J_{C F}=\right.$ $274.3 \mathrm{~Hz}), 122.6,35.4\left(\mathrm{q}, J_{C C F}=33.2 \mathrm{~Hz}\right), 25.9\left(\mathrm{~d}, J_{C C C F}=2.3 \mathrm{~Hz}\right), 14.7\left(\mathrm{~d}, J_{C C C F}=2.9\right.$ $\mathrm{Hz}) ;{ }^{19} \mathrm{~F}$ NMR (400 MHz, $\mathrm{CDCl}_{3}$ ) $\delta$-69.84; FTIR(neat): 1593, 1490, 1291, 1135, 1012, 814, 748; HRMS (EI) m/z calcd. for $\mathrm{C}_{20} \mathrm{H}_{14} \mathrm{~F}_{3} \mathrm{Br}$ : 390.0225 . Found: 390.0222 ; HPLC analysis: $98 \%$ ee (chiralcel OJ, $10.0 \% i \mathrm{Pr}-\mathrm{OH}$ in hexane, $0.8 \mathrm{~mL} / \mathrm{min} \lambda=230 \mathrm{~nm}, \mathrm{t}_{\mathrm{R}}=$ 7.5 min, major; $\mathrm{t}_{\mathrm{R}}=17.1 \mathrm{~min}$, minor), $[\alpha]_{\mathrm{D}}{ }^{25}=+137.6$ (c 2.90, $\left.\mathrm{CHCl}_{3}\right)$.

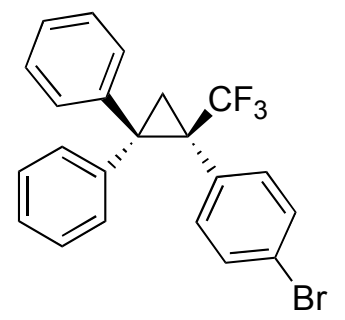

15d

(S)-(2-(4-Bromophenyl)-2-(trifluoromethyl)cyclopropane-1,1-diyl)dibenzene (15d):

The reaction was performed using $0.024 \mathrm{~g}(0.090 \mathrm{mmol})$ of the hydrazone $13 \mathrm{c}$. The crude residue was purified by column chromatography (silica gel, 20:1 v/v pentane / diethyl ether, $\left.R_{f}=0.30\right)$ to give $\mathbf{1 5 d}\left(0.028 \mathrm{~g}, 75 \%\right.$ yield). ${ }^{1} \mathrm{H}$ NMR $\left(500 \mathrm{MHz}, \mathrm{CDCl}_{3}\right) \delta 7.59$ (d, $J=7.0 \mathrm{~Hz}, 2 \mathrm{H}), 7.35-7.219(\mathrm{~m}, 8 \mathrm{H}), 7.09(\mathrm{~d}, J=9.0 \mathrm{~Hz}, 2 \mathrm{H}), 7.03-7.00(\mathrm{~m}, 2 \mathrm{H}), 6.97-$ $6.94(\mathrm{~m}, 1 \mathrm{H}), 2.37(\mathrm{dd}, J=1.0 \mathrm{~Hz}, J=6.0 \mathrm{~Hz}, 1 \mathrm{H}), 2.24(\mathrm{~d}, J=5.5 \mathrm{~Hz}, 1 \mathrm{H}) ;{ }^{13} \mathrm{C}$ NMR $\left(75.45 \mathrm{MHz}, \mathrm{CDCl}_{3}\right) \delta$ 140.7, 139.6, 133.1, 132.2, 131.1, 129.73, 129.72, 128.7, 128.4, $128.0,127.1,126.5,125.8\left(\mathrm{q}, J_{C F}=276.0 \mathrm{~Hz}\right), 122.1,39.6\left(\mathrm{q}, J_{C C F}=31.5 \mathrm{~Hz}\right) .19 .4(\mathrm{~d}$, $\left.J_{C C C F}=2.3 \mathrm{~Hz}\right) ;{ }^{19} \mathrm{~F} \mathrm{NMR}\left(400 \mathrm{MHz}, \mathrm{CDCl}_{3}\right) \delta$-61.66; FTIR(neat): 1600, 1492, 1334, 1122, 1009, 704, 695; Anal. Calcd. for $\mathrm{C}_{16} \mathrm{H}_{12} \mathrm{BrF}_{3}$ : C, 63.33; H, 3.86. Found: $\mathrm{C}, 63.04$; $\mathrm{H}, 3.88$; HPLC analysis: $99 \%$ ee (chiralcel OD, $0.5 \% i \mathrm{Pr}-\mathrm{OH}$ in hexane, $1.0 \mathrm{~mL} / \mathrm{min} \lambda=$ $230 \mathrm{~nm}, \mathrm{t}_{\mathrm{R}}=5.4 \mathrm{~min}$, minor; $\mathrm{t}_{\mathrm{R}}=7.0 \mathrm{~min}$, major $),[\alpha]_{\mathrm{D}}{ }^{25}=+161.3\left(c 1.75, \mathrm{CHCl}_{3}\right)$. 


\section{${ }^{19} \mathrm{~F}\left\{{ }^{1} \mathrm{H}\right\}$ HOESY analysis}

The cyclopropane $\mathbf{1 4 b}$ was analyzed to find its relative stereochemistry via a ${ }^{19} \mathrm{~F}\left\{{ }^{1} \mathrm{H}\right\}$ HOESY NMR experiment.

Figure S1: ${ }^{19} \mathrm{~F}\left\{{ }^{1} \mathrm{H}\right\}$ HOESY of cyclopropane $\mathbf{1 4 b}$.

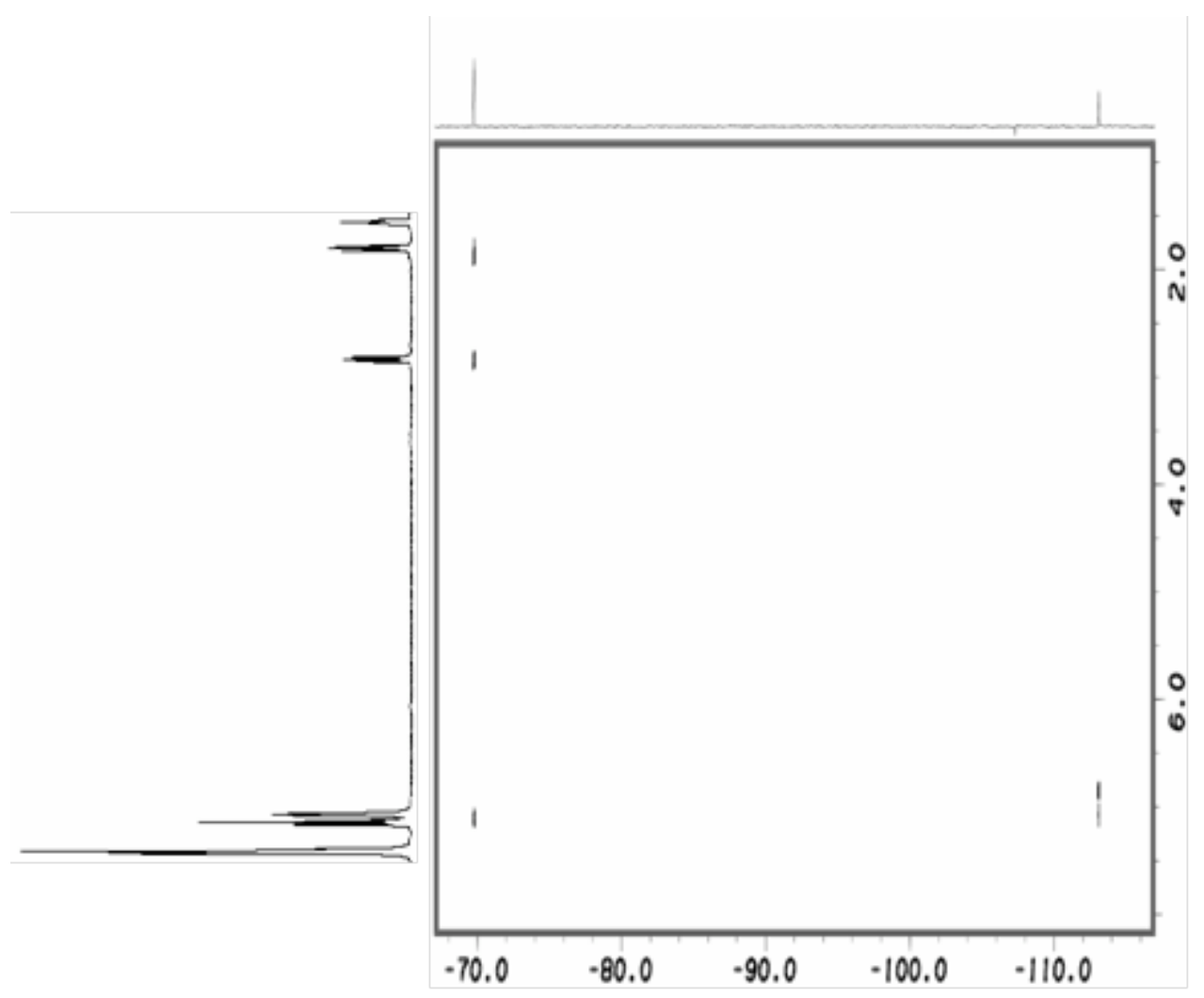

HOESY spectra were recorded using a Bruker AMX-400 spectrometer with a $\left\{{ }^{19} \mathrm{~F},{ }^{1} \mathrm{H}\right\}$ probe. The spectra were recorded in $\mathrm{CDCl}_{3}$ at $25^{\circ} \mathrm{C}$. A spectral width of $31250 \mathrm{~Hz}$ in $\mathrm{f}_{2}$ dimension $\left({ }^{19} \mathrm{~F}\right)$ and $4265 \mathrm{~Hz}$ in $\mathrm{f}_{1}$ dimension $\left({ }^{1} \mathrm{H}\right)$ were used. 128 scans were collected for each of the 128 increments, with a relaxation delay of $2.0 \mathrm{~s}$, using the phase-sensitive TPPI method. ${ }^{3}$ The spectra were processed using a $2 \mathrm{~K}$ X $2 \mathrm{~K}$ matrix, apodized using a cosine-squared function.

${ }^{3}$ C. Yu \& G. Levy, J. Am. Chem. Soc. 1984, 106, 6533. 


\section{X-ray analysis}

The cyclopropane $\mathbf{1 5 b}$ was converted to $\mathbf{1 6}$ by the following reactions in order to produce crystalline material which was suitable to grow a crystal for X-ray analysis.

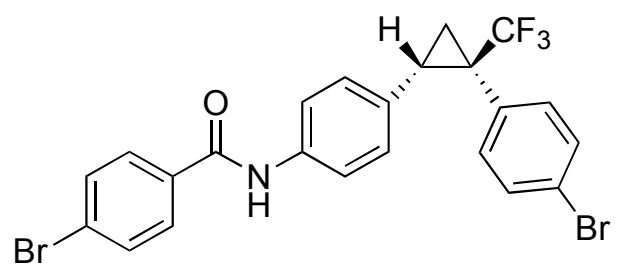

16

1-Bromo-4-((1R,2S)-2-(4-(4-bromophenylamido)phenyl)-1(trifluoromethyl)cyclopropyl)benzene (16):

Cyclopropane 15b (0.182 g, $0.471 \mathrm{mmol})$ was dissolved in EtOAc and EtOH (1:1 ratio $\mathrm{v} / \mathrm{v}, 10 \mathrm{~mL}) . \mathrm{SnCl}_{2} * 2 \mathrm{H}_{2} \mathrm{O}(0.460 \mathrm{~g}, \sim 5$ equiv) was then added in one portion. The contents was brought to reflux and stirred at reflux for $3 \mathrm{~h}$. After $3 \mathrm{~h}$, the reaction was cooled to room temperature and poured into an ice bath. Sodium bicarbonate was then added until $\mathrm{pH}$ of $\sim 8$ was obtained, then extracted with EtOAc ( 3 X $25 \mathrm{~mL}$ ). The organic layers were combined then washed with brine, dried over $\mathrm{MgSO}_{4}$, filtered, and concentrated in vacuo. The crude residue was then purified via column chromatography ( the following step.

The cyclopropyl aniline was dissolved in DCM $(10 \mathrm{~mL})$ and stirred at room temperature. Triethyl amine ( $0.06 \mathrm{~mL}, 1.10$ equiv) was added dropwise, followed by addition of $p$ bromobenzoyl chloride in one portion. The reaction was monitored by TLC for consumption of the cyclopropyl aniline. After consumption was complete $(\sim 3 \mathrm{~h})$, sat. $\mathrm{NH}_{4} \mathrm{Cl}(10 \mathrm{~mL})$ was added. The layers were then separated and the organic layer was washed with sat. $\mathrm{NH}_{4} \mathrm{Cl}(10 \mathrm{~mL})$, dried over $\mathrm{MgSO}_{4}$, filtered, and concentrated in vacuo. The crude residue was further purified by column chromatography (silica gel, pentane, $R_{f}$ $=0.26)$ to give $16(0.138 \mathrm{~g}, 55 \%$ overall yield $) . \mathrm{Mp}=200-201^{\circ} \mathrm{C}{ }^{1} \mathrm{H} \mathrm{NMR}(400 \mathrm{MHz}$, $\left.\mathrm{CDCl}_{3}\right) \delta 7.69(\mathrm{~d}, J=8.8 \mathrm{~Hz}, 3 \mathrm{H}), 7.60(\mathrm{~d}, J=8.4 \mathrm{~Hz}, 2 \mathrm{H}), 7.40(\mathrm{~d}, J=8.4 \mathrm{~Hz}, 2 \mathrm{H}), 7.31$ $(\mathrm{d}, J=8.4 \mathrm{~Hz}, 2 \mathrm{H}), 7.02(\mathrm{~d}, J=8.4 \mathrm{~Hz}, 2 \mathrm{H}), 6.79(\mathrm{~d}, J=8.4 \mathrm{~Hz}, 2 \mathrm{H}), 2.85(\mathrm{dd}, J=7.2$ $\mathrm{Hz}, J=9.6 \mathrm{~Hz}, 1 \mathrm{H}), 1.89(\mathrm{dd}, J=6.4 \mathrm{~Hz}, J=9.2 \mathrm{~Hz}, 1 \mathrm{H}), 1.65-1.62(\mathrm{~m}, 1 \mathrm{H}) ;{ }^{13} \mathrm{C} \mathrm{NMR}$ $\left(75.45 \mathrm{MHz}, \mathrm{CDCl}_{3}\right) \delta 164.7,136.4,134.0,133.6,131.9,131.5,131.3,130.6,128.6$, $128.5,126.6,125.5\left(\mathrm{q}, J_{C F}=274.3 \mathrm{~Hz}\right), 122.7,119.8,35.2\left(\mathrm{q}, J_{C C F}=32.6 \mathrm{~Hz}\right), 25.3,14.6$; ${ }^{19} \mathrm{~F} \mathrm{NMR}\left(400 \mathrm{MHz}, \mathrm{CDCl}_{3}\right) \delta-69.81$; FTIR(neat): 1648, 1523, 1153, 1131, 815, 699; HRMS (EI) $\mathrm{m} / \mathrm{z}$ calcd. for $\mathrm{C}_{23} \mathrm{H}_{16} \mathrm{~F}_{3} \mathrm{NOBr}_{2}$ : 536.9545. Found: 536.9554; HPLC analysis: $>99 \%$ ee (chiralcel OJ, $10 \% \mathrm{Pr}-\mathrm{OH}$ in hexane, $0.8 \mathrm{~mL} / \mathrm{min} \lambda=230 \mathrm{~nm}, \mathrm{t}_{\mathrm{R}}=33.0 \mathrm{~min}$, major; $\mathrm{t}_{\mathrm{R}}=46.4$ min, minor $),[\alpha]_{\mathrm{D}}{ }^{25}=+130.0\left(\right.$ c $\left.1.10, \mathrm{CHCl}_{3}\right)$.

Crystalline material of $\mathbf{1 6}$ suitable for X-ray analysis was grown as follows: In a $4 \mathrm{~mL}$ vial $50 \mathrm{mg}$ of 16 was dissolved in THF $(\sim 1 \mathrm{~mL})$. The uncapped vial was then placed inside a larger $20 \mathrm{~mL}$ vial which contained $5 \mathrm{~mL}$ of pentane. The larger vial was then capped and the pentane vapors were allowed to slowly diffuse into the smaller vial for 5 days at which time crystalline material of $\mathbf{1 6}$ suitable for X-ray analysis were obtained. 
Figure S2: X-ray structure of compound $16^{4}$

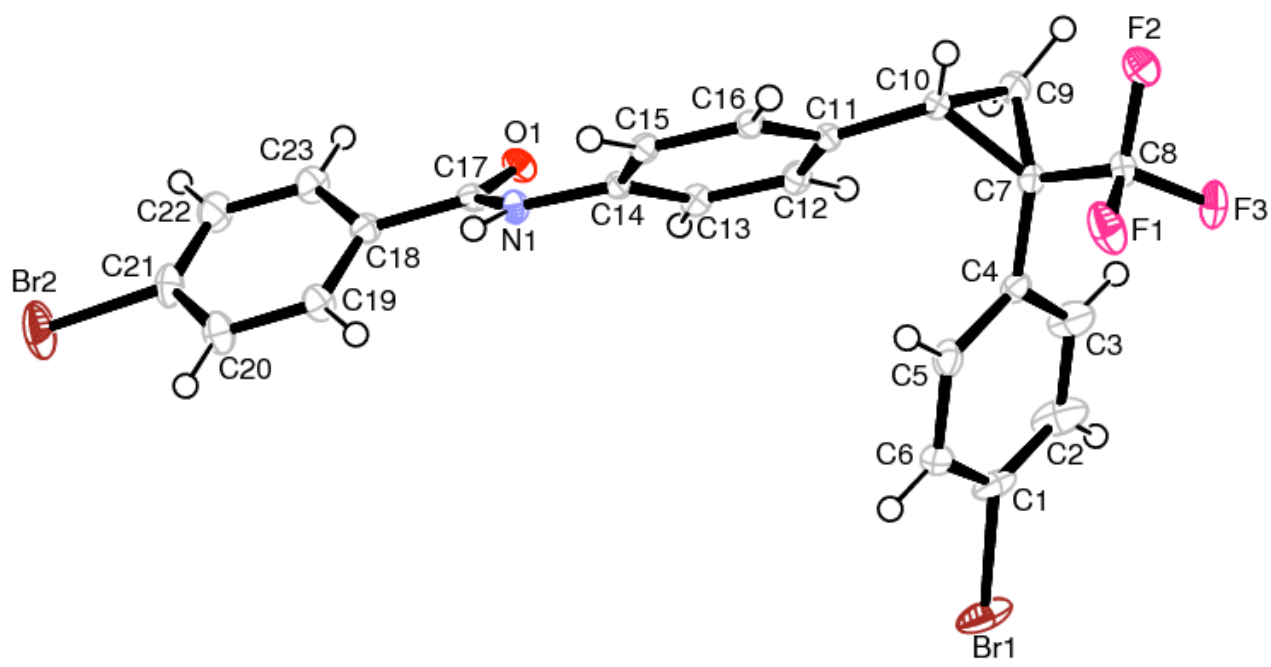

Fig. S2. ORTEP plot and label representation of cyclopropane 16. Displacement ellipsoids are drawn at the $50 \%$ probability level.

\footnotetext{
${ }^{4}$ The X-ray crystallographic data has been submitted to the Cambridge Structure Database: Pitak, M. and Coppens, P. Private Communication, 2007, CCDC 638557.
} 


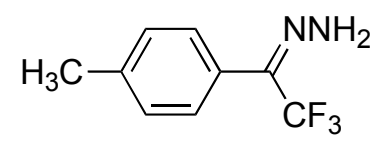

$13 a$
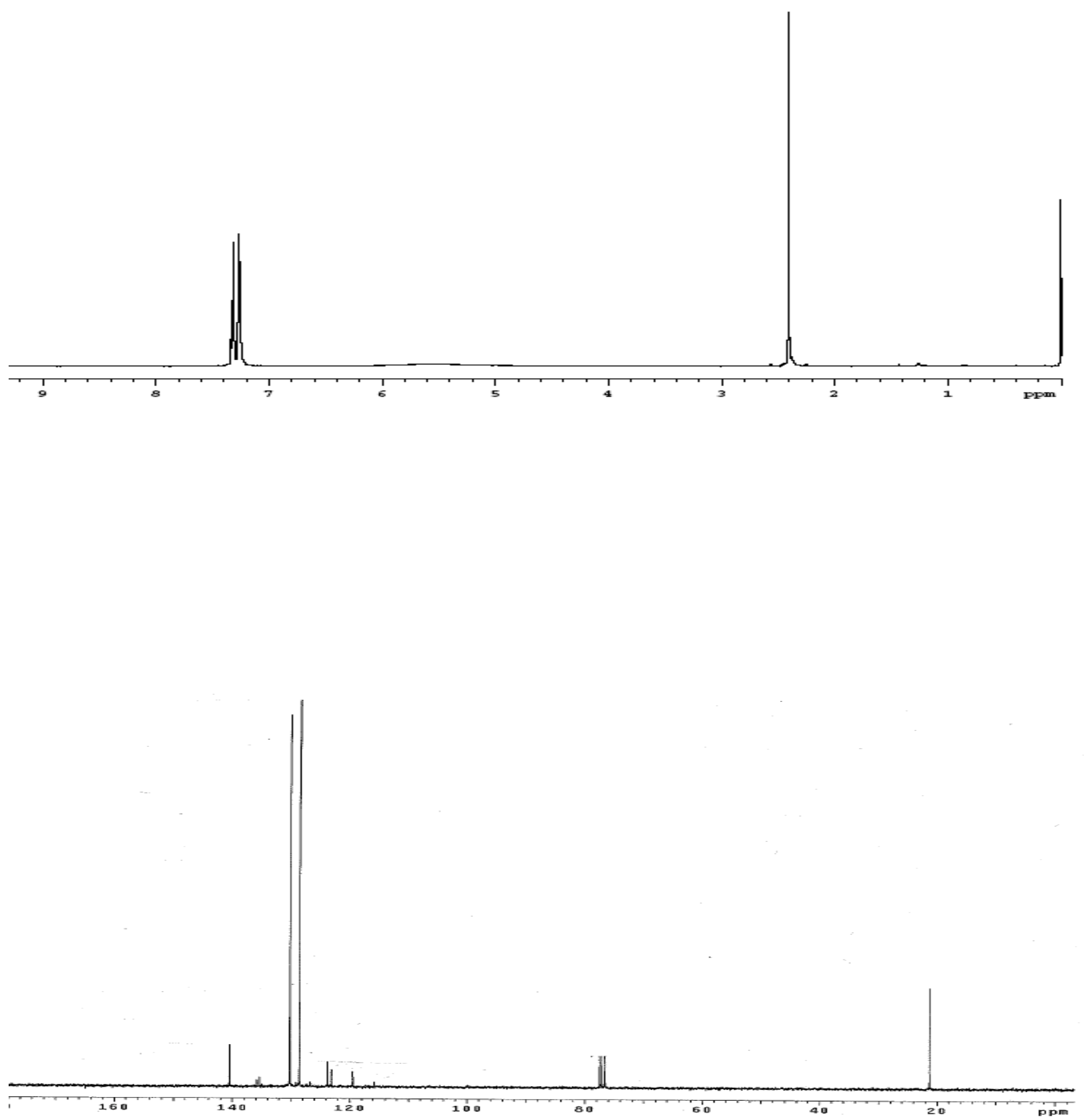

S-15 


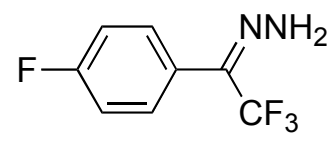

13b
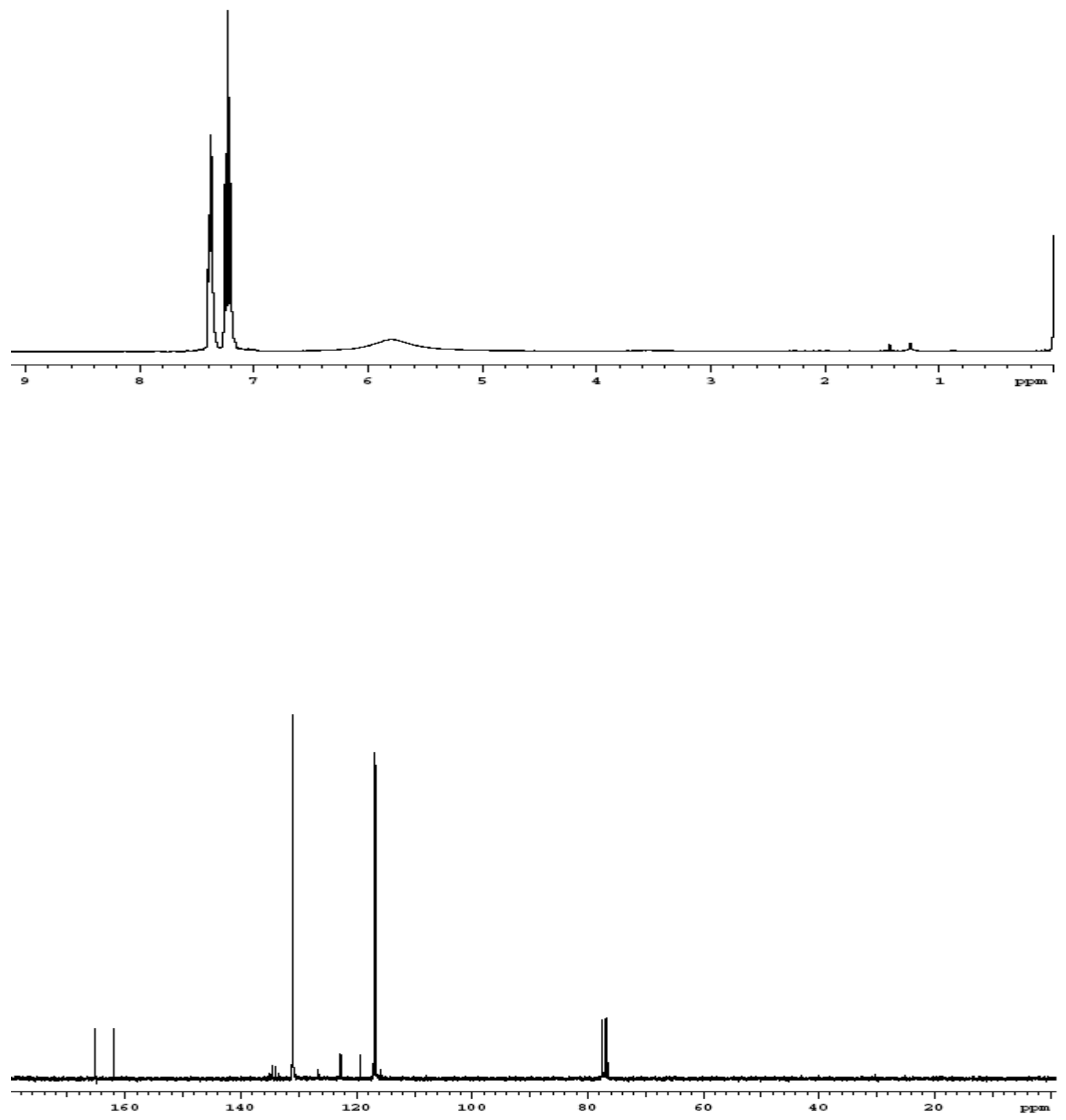

S-16 


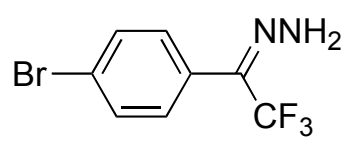

$13 c$
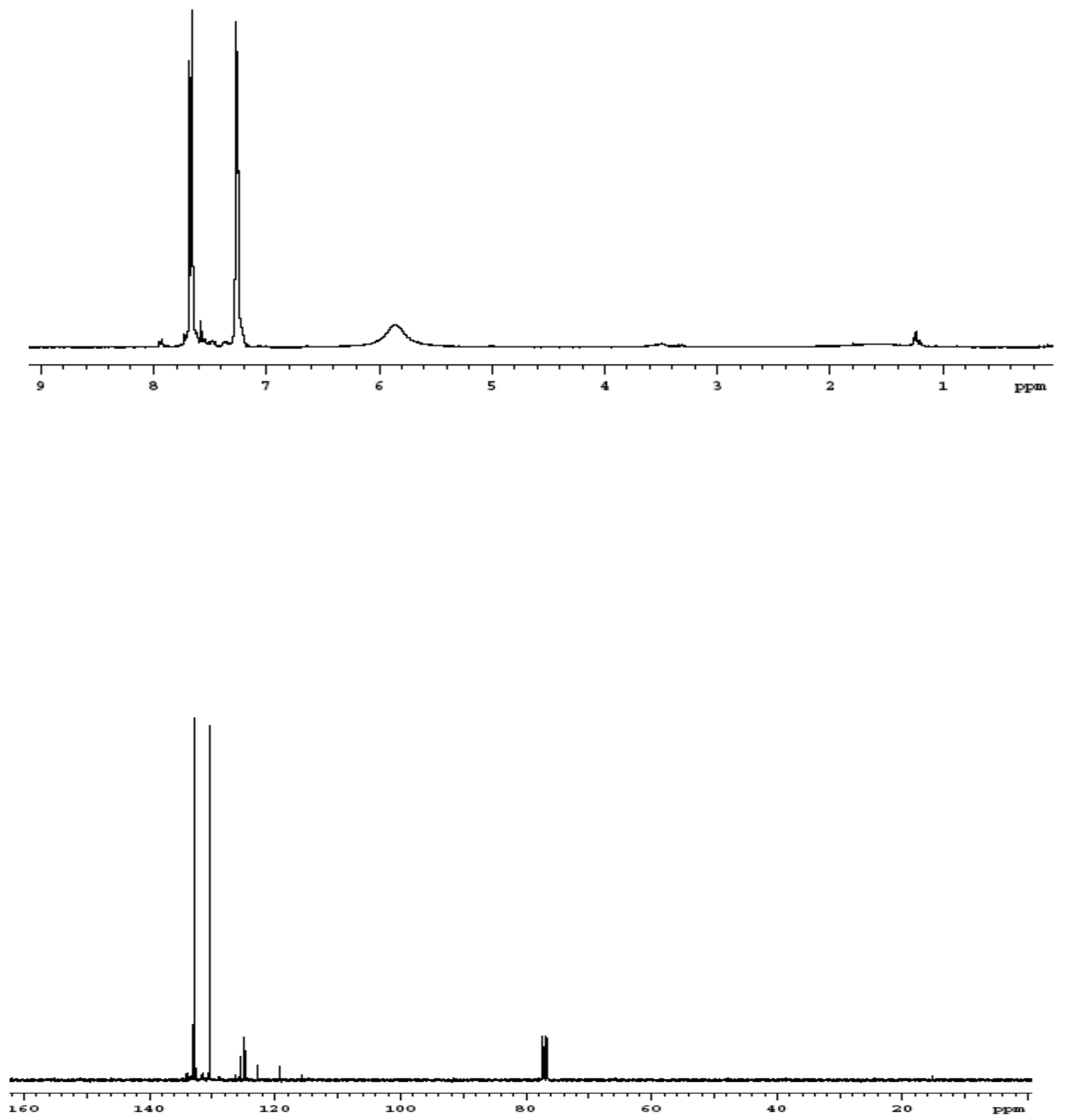

S-17 


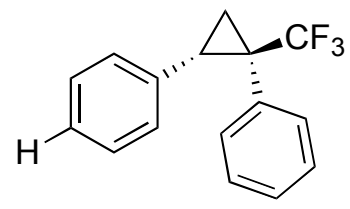

$10 a$
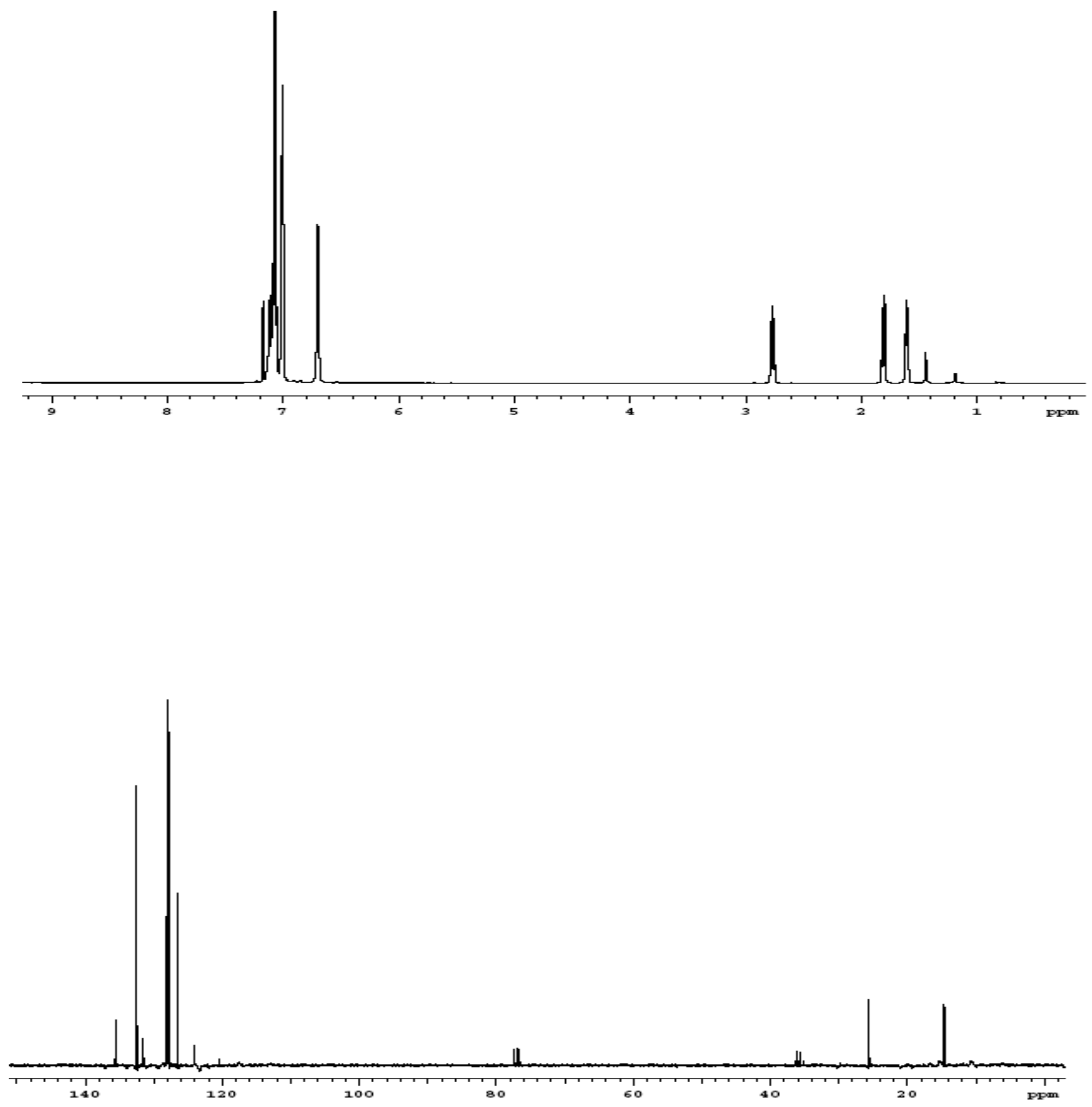


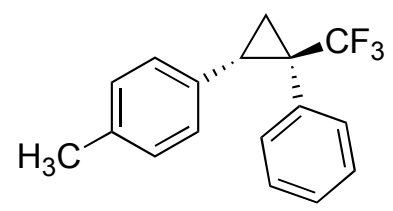

10b
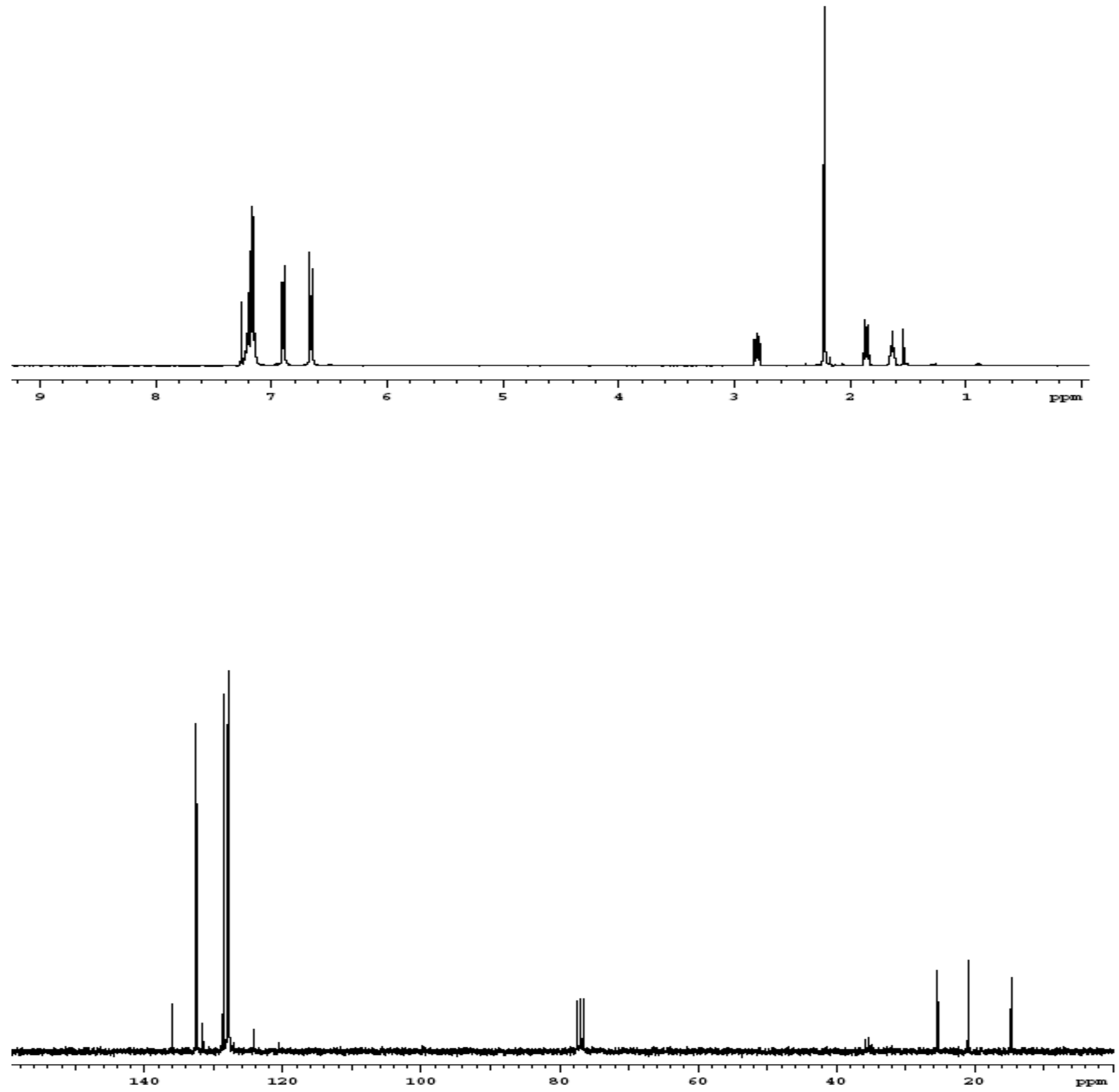

S-19 


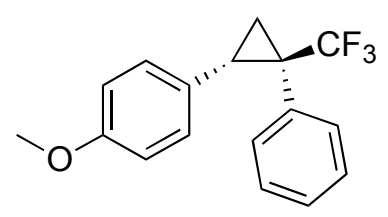

10c
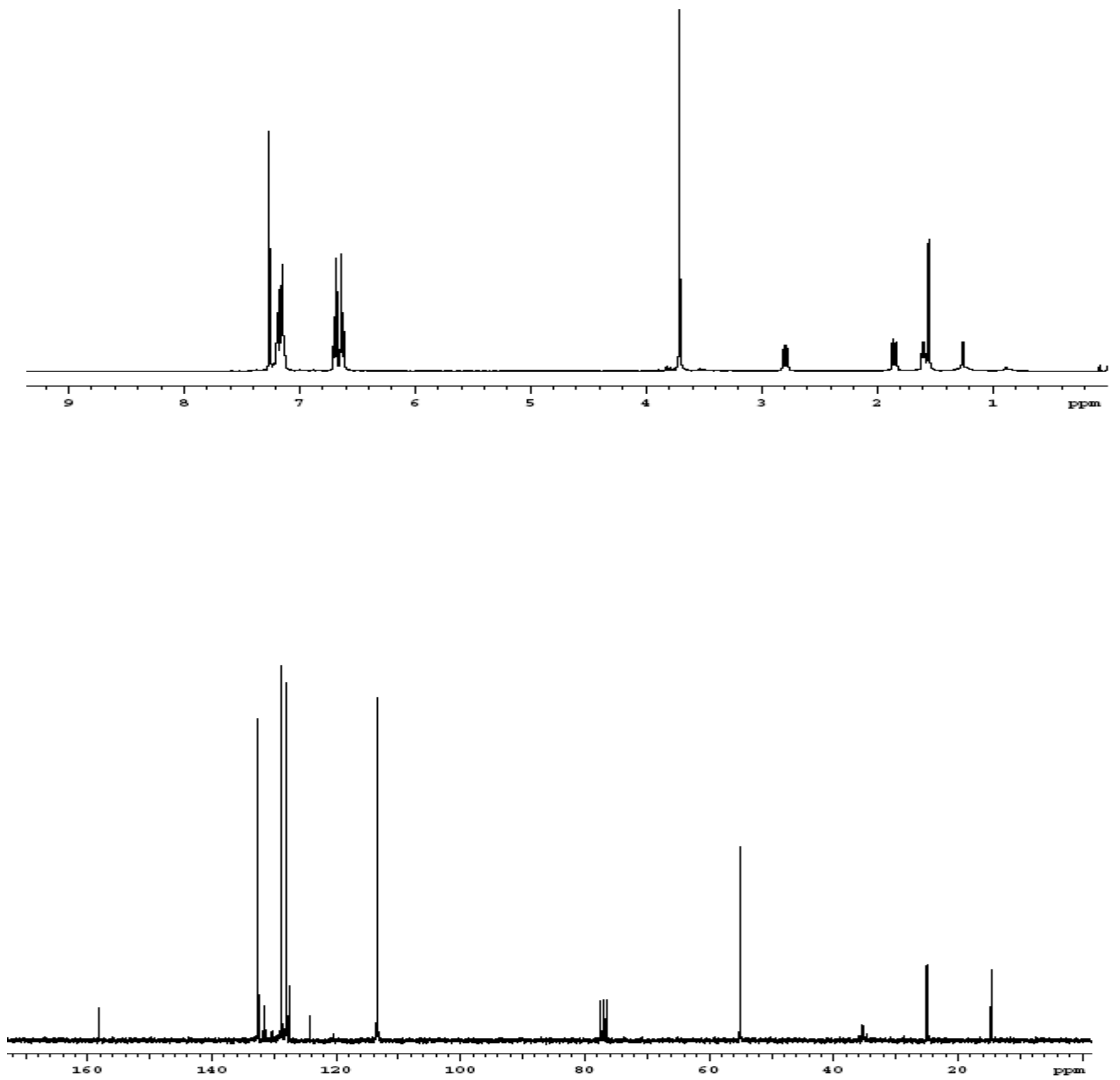
<smiles>FC(F)(F)C1(c2ccccc2)C[C@H]1c1ccc(Cl)cc1</smiles>

10d
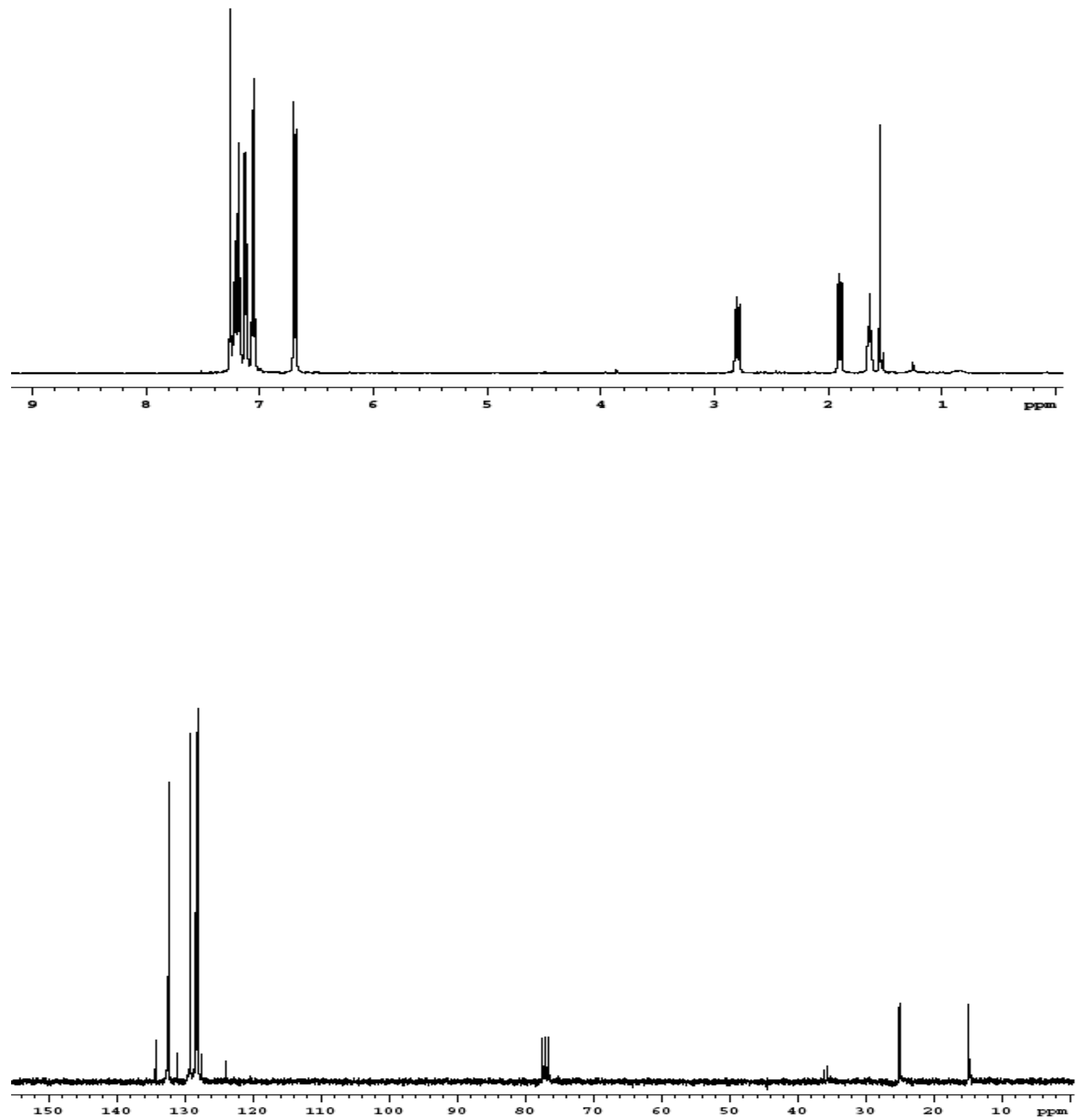


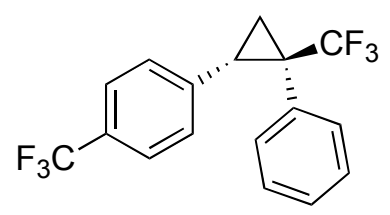

$10 \mathrm{e}$
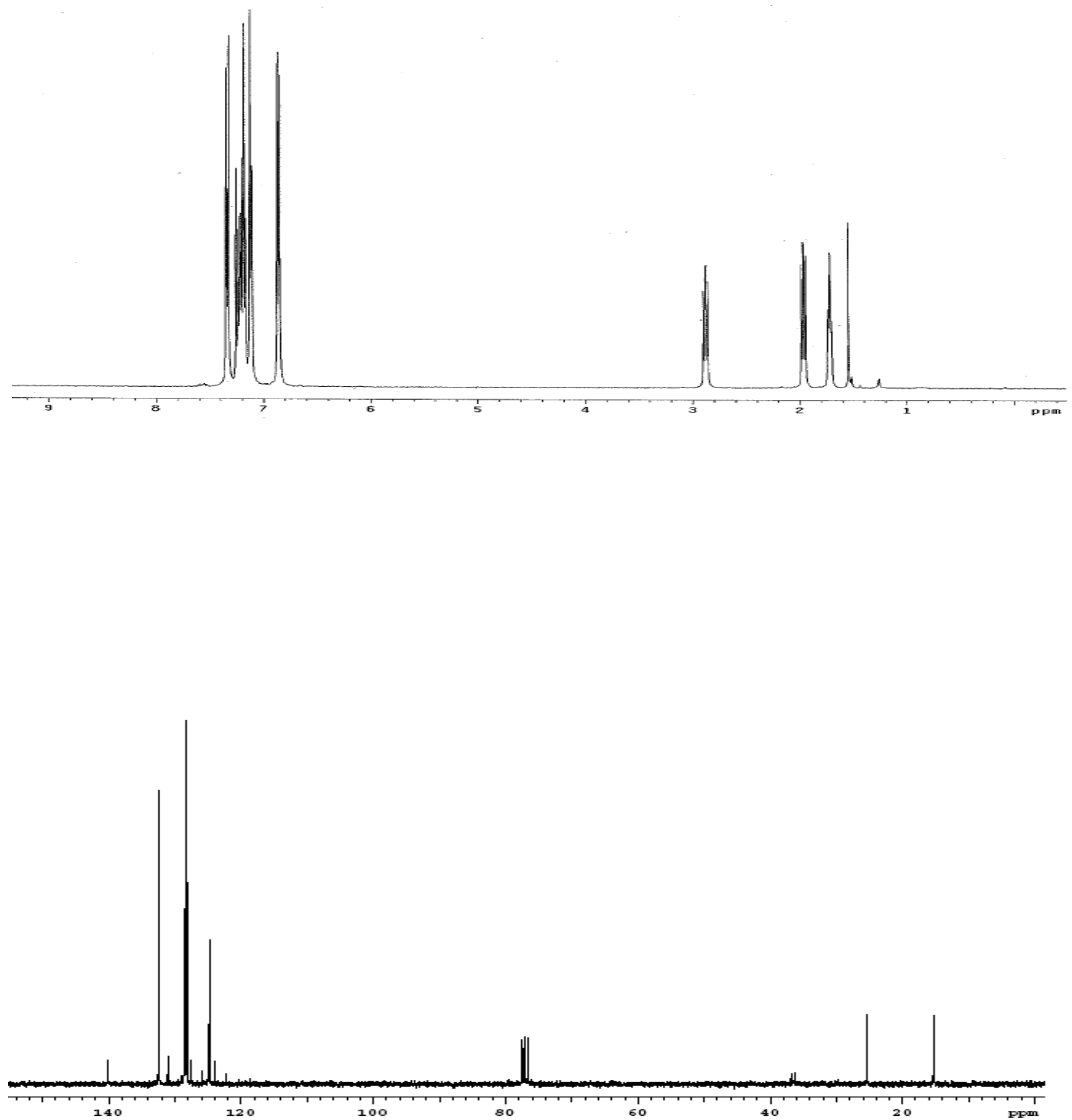

S-22 


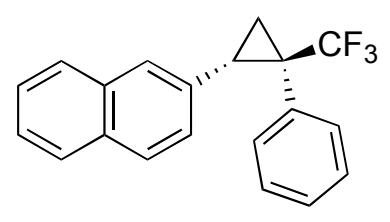

$10 f$


S-23 


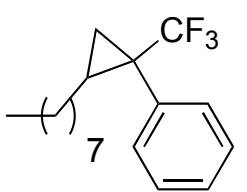

$10 \mathrm{~g}$
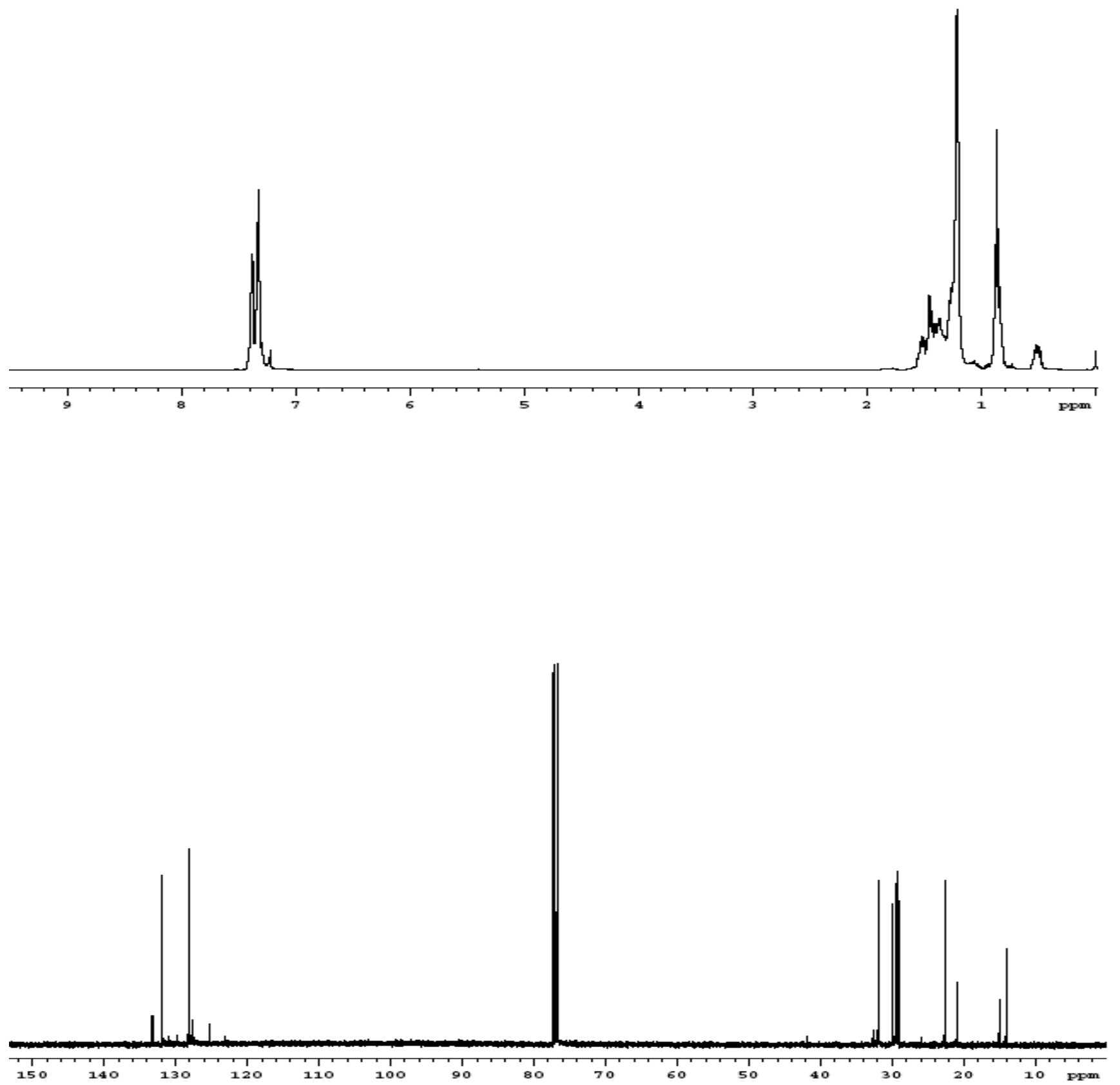


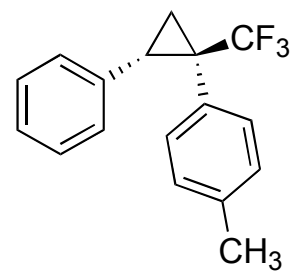

$14 a$
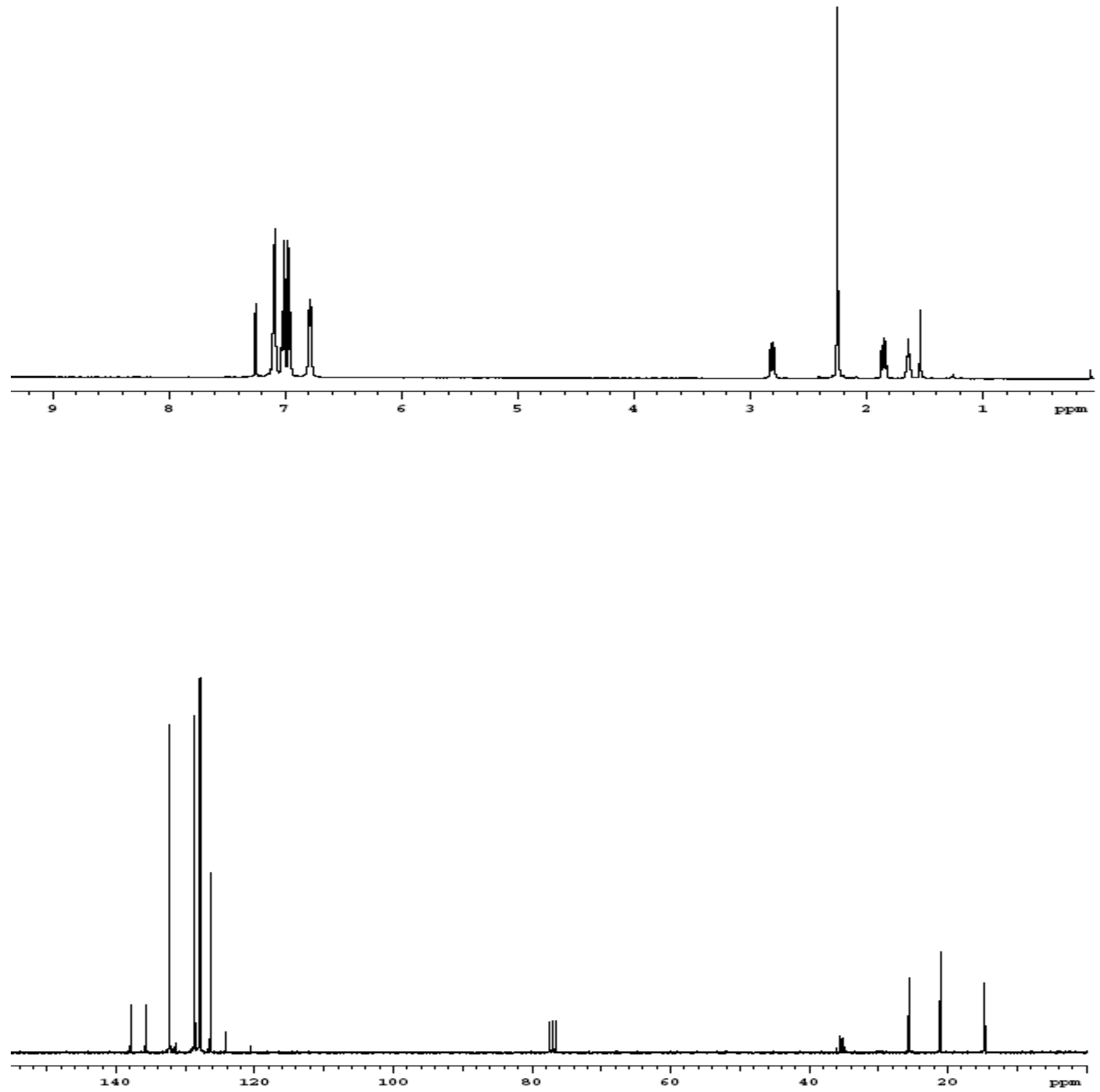


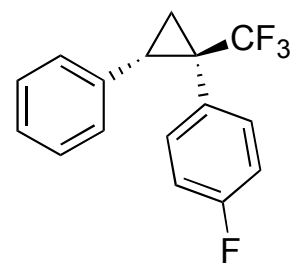

14b
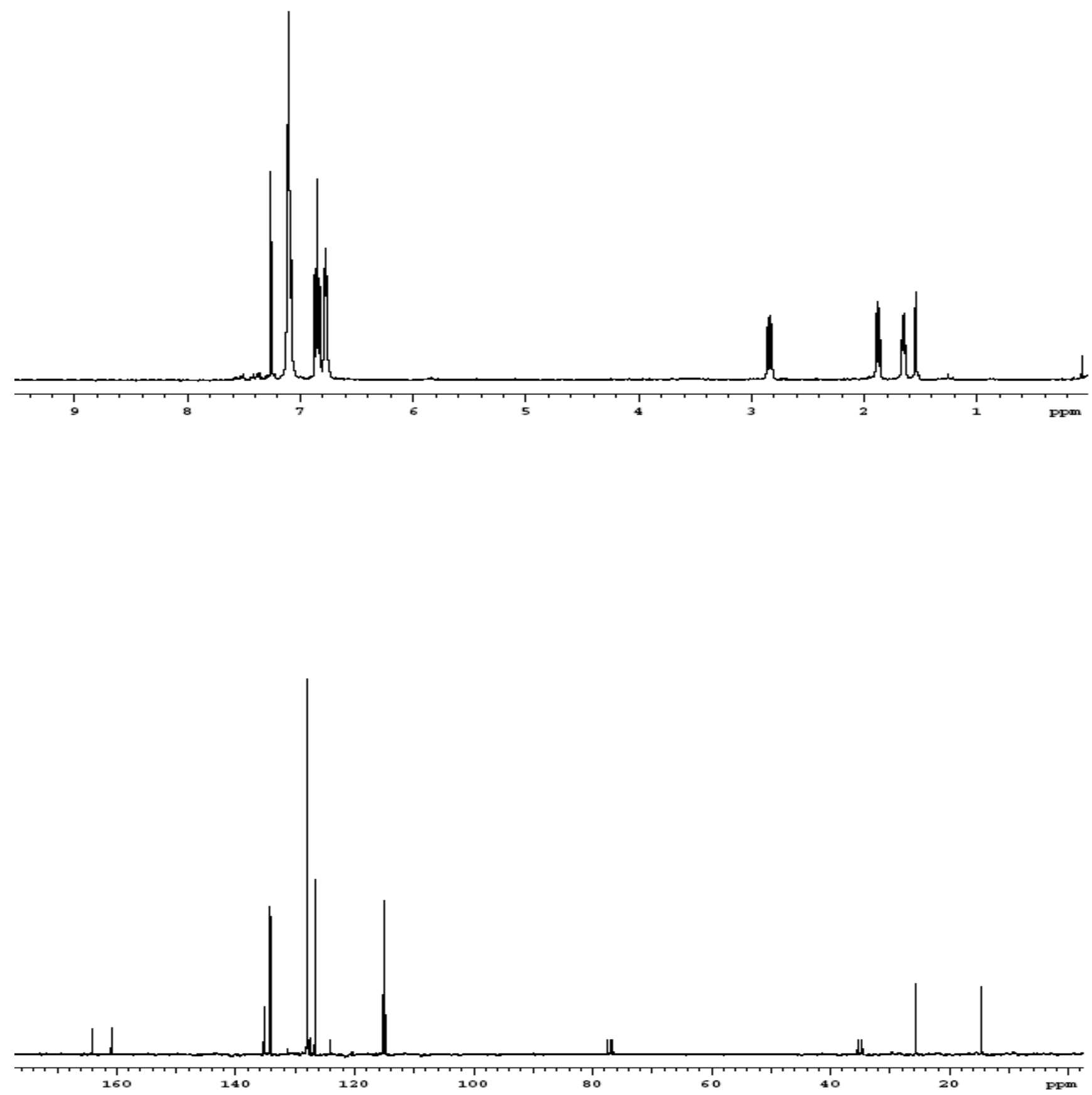


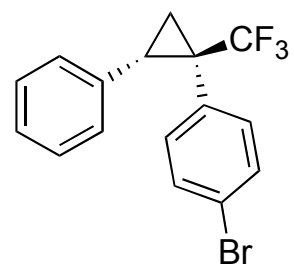

$14 c$
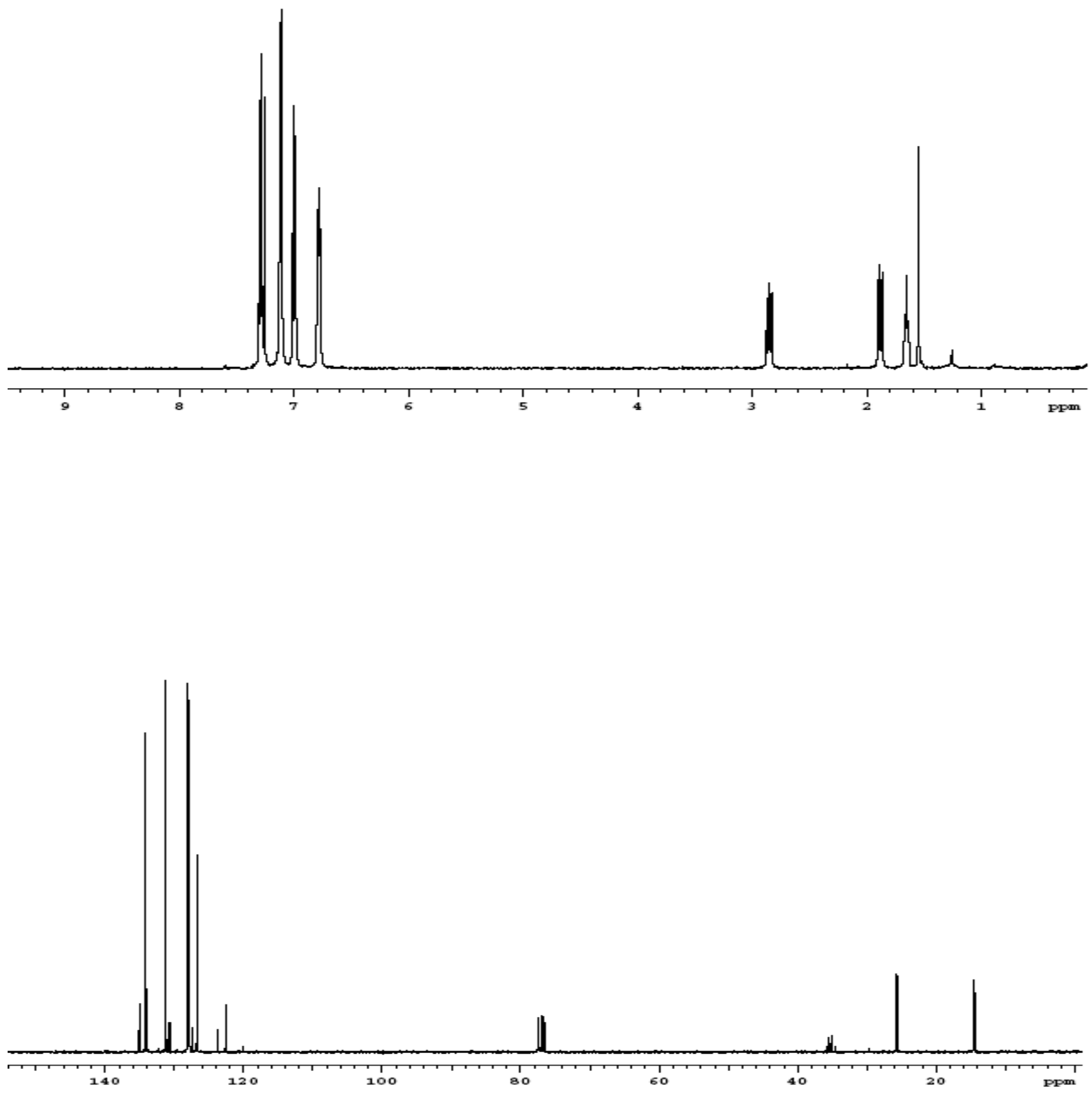

S-27 


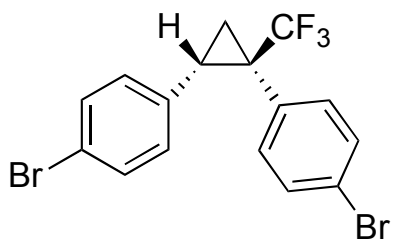

$15 a$
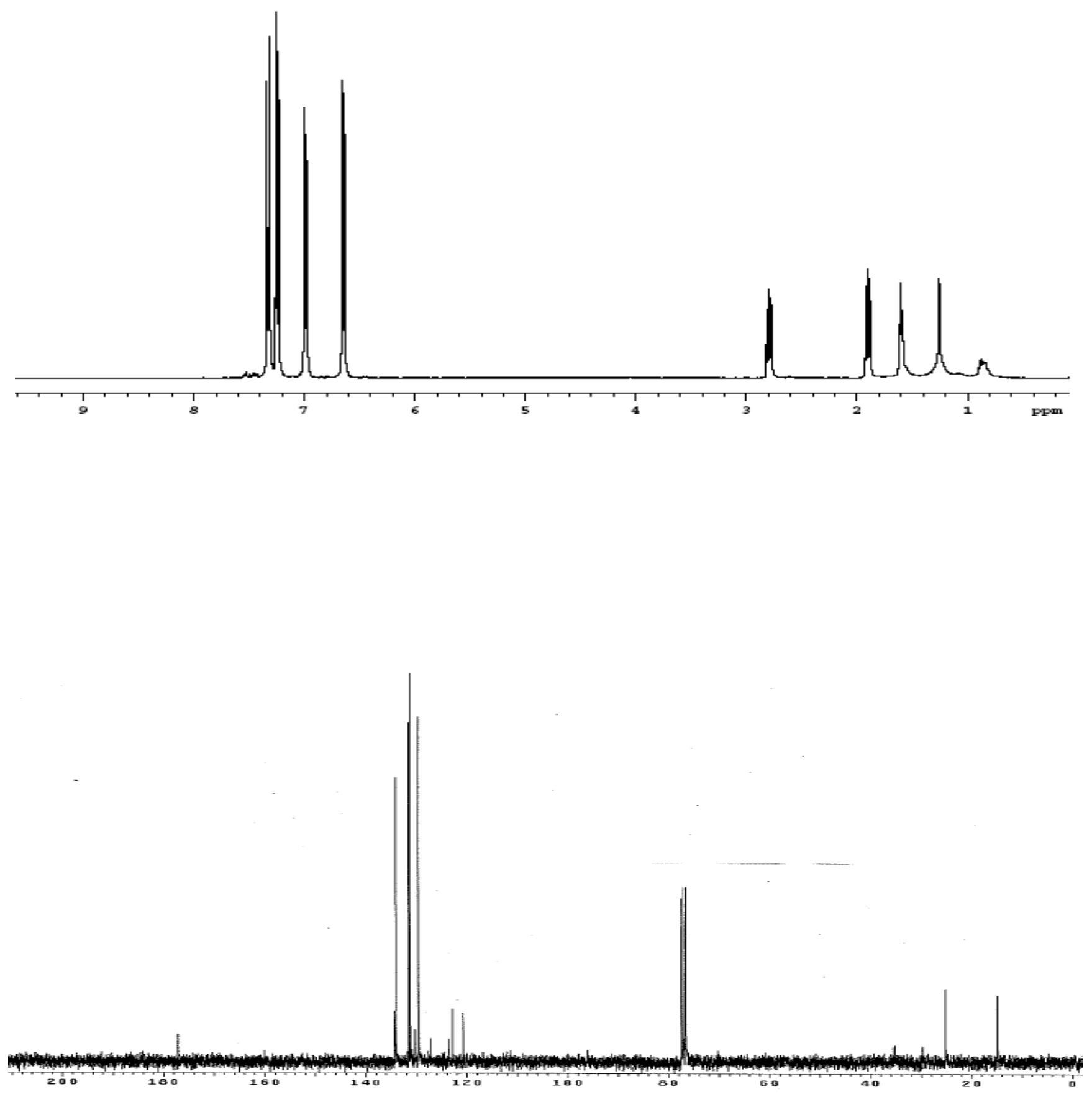


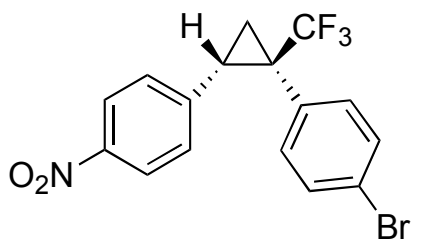

15b
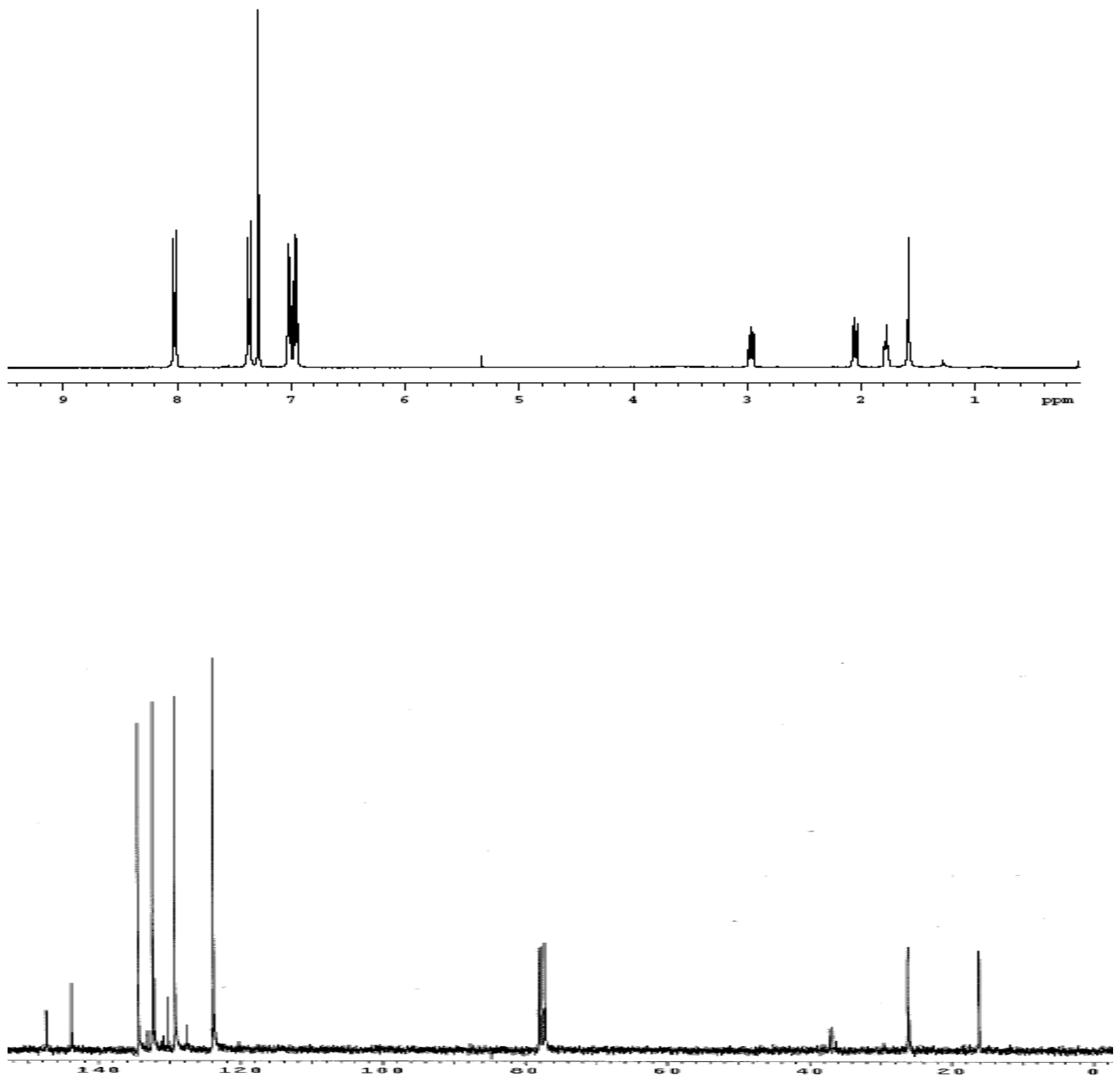

S-29 


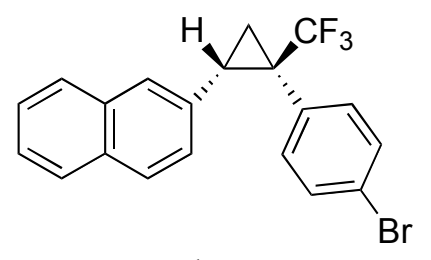

$15 c$
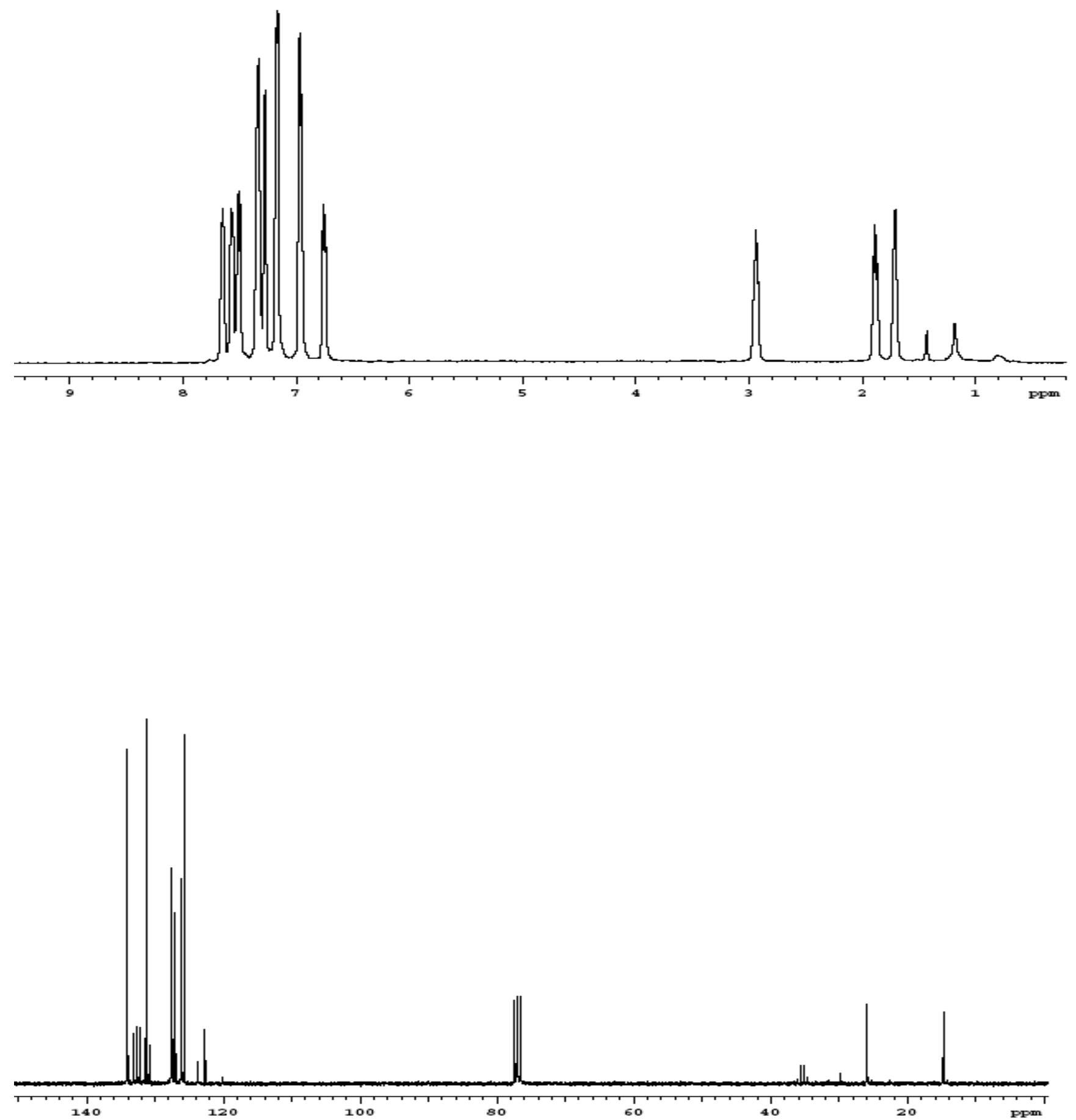

S-30 


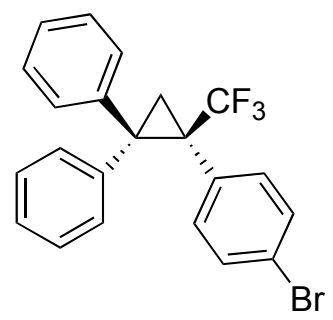

15d
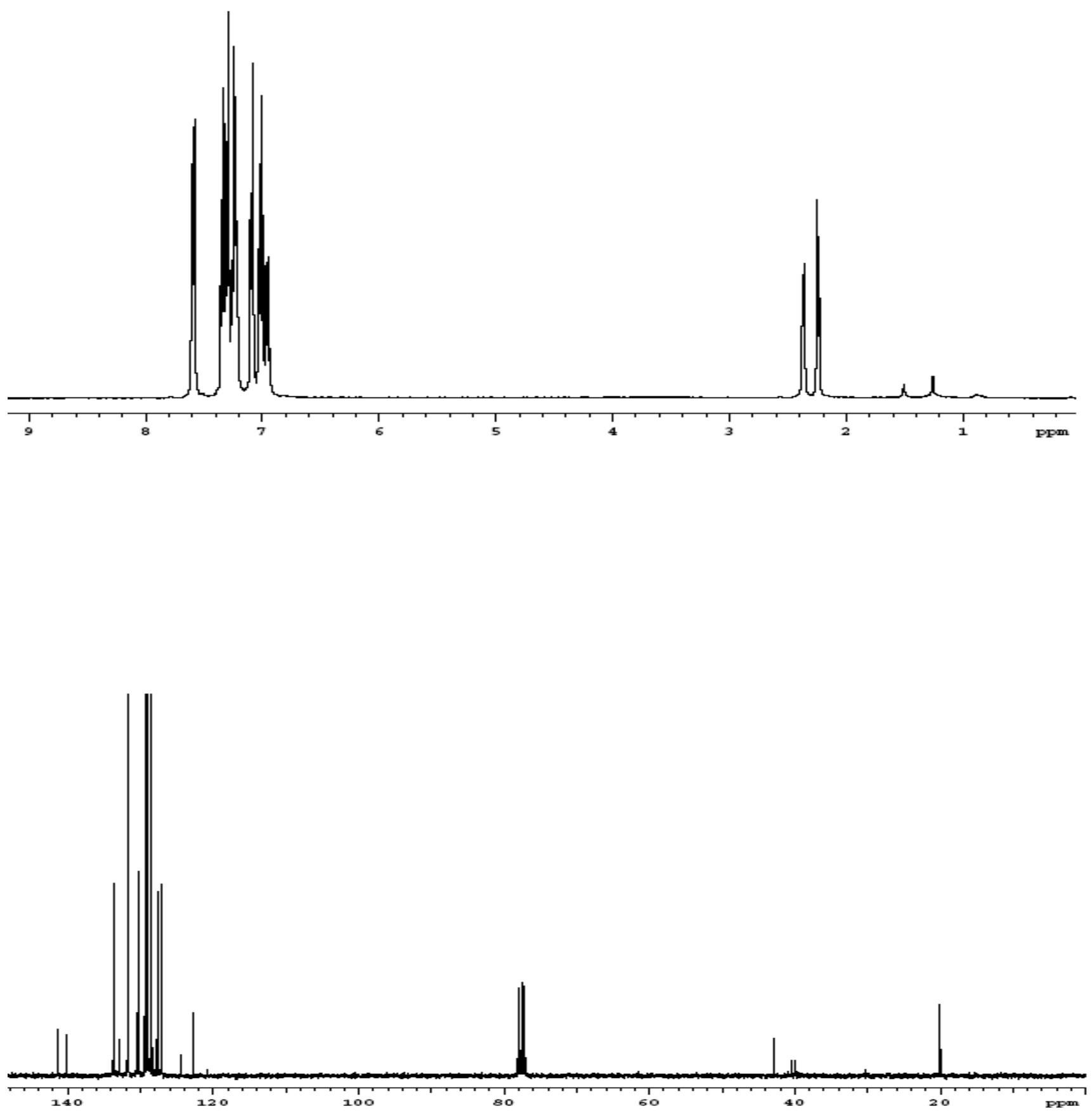

S-31 


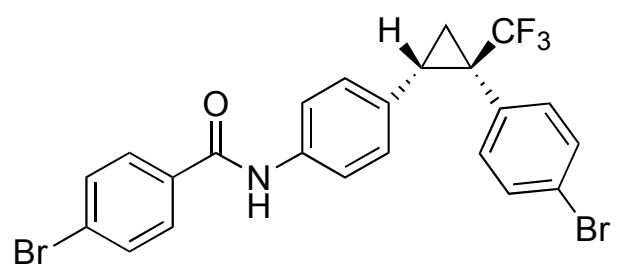

16
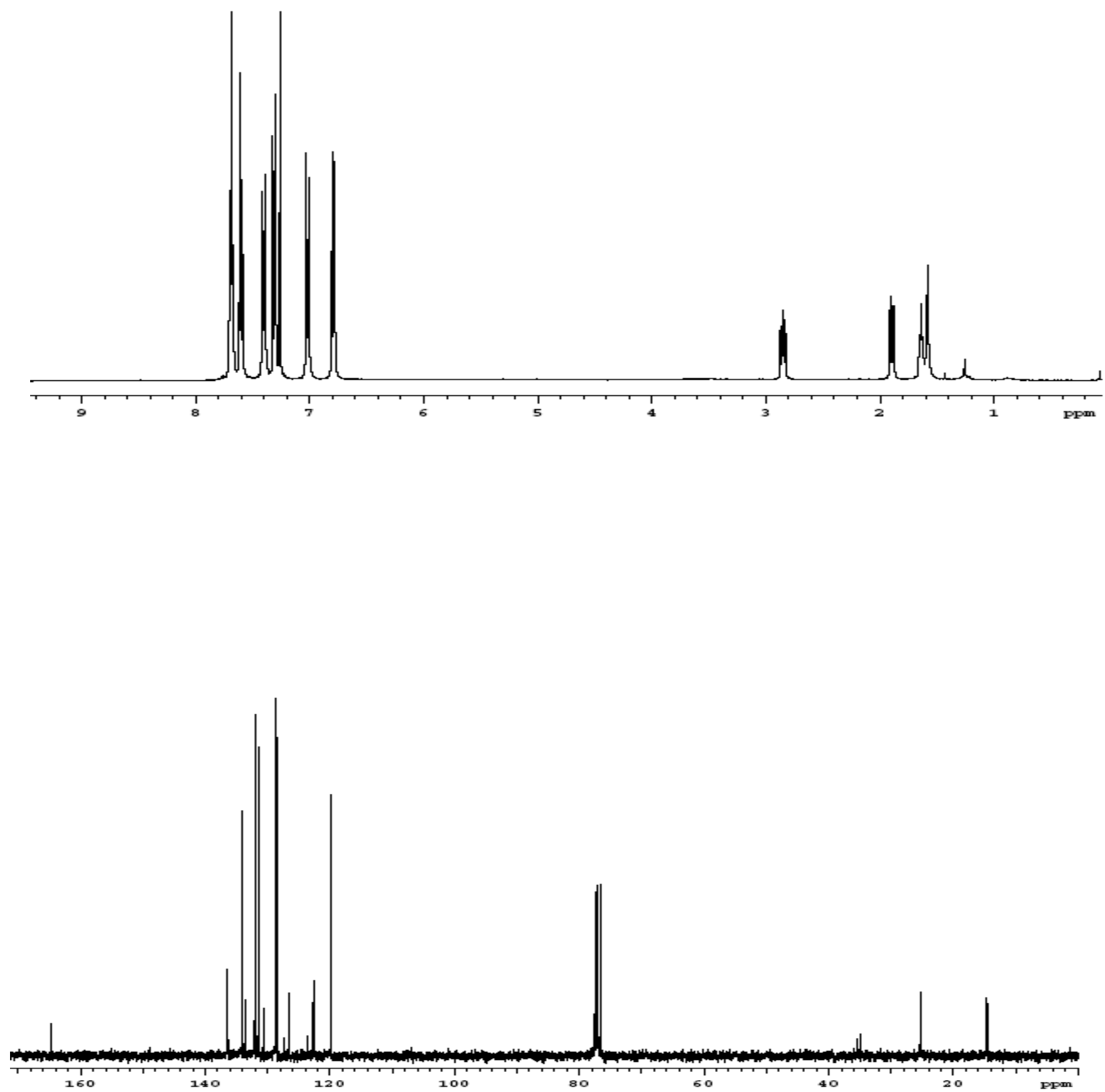

S-32 